MATHEMATICS OF COMPUTATION

Volume 77, Number 263, July 2008, Pages 1653-1679

S 0025-5718(08)02073-5

Article electronically published on February 4, 2008

\title{
THE HYPERDETERMINANT AND TRIANGULATIONS OF THE 4-CUBE
}

\author{
PETER HUGGINS, BERND STURMFELS, JOSEPHINE YU, AND DEBBIE S. YUSTER
}

\begin{abstract}
The hyperdeterminant of format $2 \times 2 \times 2 \times 2$ is a polynomial of degree 24 in 16 unknowns which has 2894276 terms. We compute the Newton polytope of this polynomial and the secondary polytope of the 4-cube. The 87959448 regular triangulations of the 4 -cube are classified into $25448 \mathrm{D}$ equivalence classes, one for each vertex of the Newton polytope. The 4-cube has 80876 coarsest regular subdivisions, one for each facet of the secondary polytope, but only 268 of them come from the hyperdeterminant.
\end{abstract}

\section{INTRODUCTION}

The hyperdeterminant is a fundamental object of multilinear algebra. We recall its definition from the book by Gel'fand, Kapranov, and Zelevinsky [12, which is our basic reference. Given an $n$-linear form with unknown coefficients,

$$
F(x)=\sum_{i_{1}=1}^{r_{1}} \sum_{i_{2}=1}^{r_{2}} \cdots \sum_{i_{n}=1}^{r_{n}} c_{i_{1} i_{2} \cdots i_{n}} \cdot x_{i_{1}}^{(1)} x_{i_{2}}^{(2)} \cdots x_{i_{n}}^{(n)}
$$

one considers the set of all tensors $\left(c_{i_{1} i_{2} \cdots i_{n}}\right)$ in $\mathbb{C}^{r_{1}} \otimes \mathbb{C}^{r_{2}} \otimes \cdots \otimes \mathbb{C}^{r_{n}}$ such that the system of $(n-1)$-linear equations obtained by taking partial derivatives,

$$
\frac{\partial F}{\partial x_{k}^{(j)}}(x)=0 \quad \text { for } j=1,2 \ldots, n \text { and } k=1, \ldots, r_{j},
$$

has a non-trivial solution $\left(x^{(1)}, x^{(2)}, \ldots, x^{(n)}\right)$. Here non-trivial means that $x^{(i)}$ is a non-zero vector in $\mathbb{C}^{r_{i}}$. This set is an irreducible algebraic variety in $\mathbb{C}^{r_{1}} \otimes \cdots \otimes \mathbb{C}^{r_{n}}$. For many values of $r_{1}, r_{2}, \ldots, r_{n}$, this variety has codimension one, so it is the zero set of a unique (up to sign) irreducible polynomial in the unknowns $c_{i_{1} i_{2} \cdots i_{n}}$. That unique polynomial is the hyperdeterminant $D_{r_{1} r_{2} \cdots r_{n}}$.

Hyperdeterminants have numerous applications ranging from quantum information theory [14, 15 to computational biology 1, 21 and numerical analysis 17, 22. Of particular interest is the binary case, when $r_{1}=r_{2}=\cdots=r_{n}=2$. The first instance $(n=2)$ is the familiar determinant of a $2 \times 2$-matrix:

$$
D_{22}=c_{00} c_{11}-c_{01} c_{10} .
$$

Received by the editor February 9, 2006 and, in revised form, January 6, 2007.

2000 Mathematics Subject Classification. Primary 52B55; Secondary 68W30.

(C)2008 American Mathematical Society 
Next, the hyperdeterminant of a $2 \times 2 \times 2$-tensor is the following irreducible polynomial in 8 unknowns which is the sum of 12 terms of degree four:

$$
\begin{aligned}
& D_{222}=4 c_{000} c_{011} c_{101} c_{110}+4 c_{001} c_{010} c_{100} c_{111} \\
& +c_{000}^{2} c_{111}^{2}+c_{001}^{2} c_{110}^{2}+c_{010}^{2} c_{101}^{2}+c_{011}^{2} c_{100}^{2} \\
& -2 c_{000} c_{001} c_{110} c_{111}-2 c_{000} c_{010} c_{101} c_{111}-2 c_{000} c_{011} c_{100} c_{111} \\
& -2 c_{001} c_{010} c_{101} c_{110}-2 c_{001} c_{011} c_{110} c_{100}-2 c_{010} c_{011} c_{101} c_{100} \text {. }
\end{aligned}
$$

This hyperdeterminant is also known as the tangle in the physics literature.

The present paper is concerned with the case $n=4$, the next instance after the tangle. We resolve the following challenge problem which was stated by I. M. Gel'fand in his fall 2005 research seminar at Rutgers University: find the monomial expansion of the hyperdeterminant $D_{2222}$ of a $2 \times 2 \times 2 \times 2$-tensor.

Back in 1852, Schläfli had given a nested formula [12, §14.4] for $D_{2222}$, which is a polynomial of degree 24 in 16 unknowns $c_{0000}, c_{0001}, \ldots, c_{1111}$, and, 150 years later, Luque and Thibon [15] expressed $D_{2222}$ in terms of the fundamental tensor invariants. Gel'fand's challenge to the computer algebra community was to expand these formulas into monomials. We obtained the following result:

Theorem 1. The hyperdeterminant $D_{2222}$ is the sum of 2894276 monomials in 9617 orbits. The Newton polytope of $D_{2222}$ is an 11-dimensional polytope with 268 facets in 8 orbits and 25448 vertices in 111 orbits. It contains the exponent vectors of 20992 monomials in 69 orbits which do not appear in $D_{2222}$.

In this theorem, the term orbits refers to the full symmetry group $B_{4}$ of the 4 -dimensional cube, which has order 384 , and the Newton polytope is the convex hull in $\mathbb{R}^{16}$ of the exponent vectors of all monomials appearing in $D_{2222}$.

Our computational proof of Theorem 1 is explained in Sections 3 and 4. We list representatives for each orbit of facets, vertices, and "missing monomials," and we discuss key properties found in these data. Complete data are available at our supplementary materials website bio.math.berkeley.edu/4cube/.

Gel'fand, Kapranov, and Zelevinsky [12, §11] found a beautiful and deep relationship between the monomials in the $2 \times 2 \times \cdots \times 2$-hyperdeterminant and the triangulations of the $n$-dimensional cube. In the language of polyhedral geometry, this relationship can be stated as follows: the secondary polytope of the $n$-cube equals the Minkowski sum of the Newton polytopes of the hyperdeterminants corresponding to all the faces of the $n$-cube, up to dimension $n$. Readers unfamiliar with these geometric concepts will find an elementary and self-contained introduction in Section 2, where we explain the correspondence between the monomials in $D_{222}$ and the triangulations of the 3 -cube.

Theorem 1 opened up the possibility of determining the same correspondence for the 4-cube. However, we found the computational challenges to be formidable, in light of the size of the secondary polytope of the 4-cube:

Theorem 2. The 4-cube has 87959448 regular triangulations in 235277 orbits. The secondary polytope of the 4-cube has 80876 facets in 334 orbits.

The first statement in Theorem 2 is a known result due to Pfeifle and Rambau [16], who had computed all regular triangulations of the 4-cube using Rambau's software TOPCOM [17. We independently verified their enumeration. The second statement in Theorem 2 is one of the contributions of this paper. 
Equipped with Theorems 1 and 2, it took us two more months of serious computational work to obtain what we consider to be the main mathematical result of this paper: the extension of the material in Section 2 from dimension three to dimension four. Each regular triangulation of the 4-cube maps to a monomial in $D_{2222}$, and the fibers of this map are the D-equivalence classes of [12. We found that, up to symmetry, there are $111 D$-equivalence classes. They are discussed in Section 6. Section 7 studies the 80876 facets in Theorem 2, and it classifies the coarsest regular polyhedral subdivisions of the 4-cube. Most of the algorithms and software we developed and used here can easily be generalized and applied to other polynomials and polytopes with symmetry.

Section 5 places our computations into a larger mathematical context. The hyperdeterminant $D_{2222}$ is the $A$-discriminant for the matrix of the 4 -cube

$$
A=\left[\begin{array}{llllllllllllllll}
1 & 1 & 1 & 1 & 1 & 1 & 1 & 1 & 1 & 1 & 1 & 1 & 1 & 1 & 1 & 1 \\
0 & 0 & 0 & 0 & 0 & 0 & 0 & 0 & 1 & 1 & 1 & 1 & 1 & 1 & 1 & 1 \\
0 & 0 & 0 & 0 & 1 & 1 & 1 & 1 & 0 & 0 & 0 & 0 & 1 & 1 & 1 & 1 \\
0 & 0 & 1 & 1 & 0 & 0 & 1 & 1 & 0 & 0 & 1 & 1 & 0 & 0 & 1 & 1 \\
0 & 1 & 0 & 1 & 0 & 1 & 0 & 1 & 0 & 1 & 0 & 1 & 0 & 1 & 0 & 1
\end{array}\right] .
$$

Dickenstein, Feichtner, and Sturmfels $[8$ recently gave a recipe for computing the Newton polytope of the $A$-discriminant for any point configuration $A$. Our computations are complementary to their tropical approach. We represent subdivisions of the cube $A$ by their dual polyhedral complexes, here called tight spans as in [13]. The related cases when $A$ is a second hypersimplex or a product of two simplices correspond to finite metrics [20] and to tropical convexity [9], respectively. We also study the irreducible factorizations of all leading forms of $D_{2222}$ that are supported on facets of the Newton polytope.

Summarizing our discussion in this introduction, we wish to highlight the following three contributions to the mathematics of computation made here:

- solution to a computational challenge problem posed by I.M. Gel'fand,

- new theorems in algebra (hyperdeterminant) and geometry (4-cube),

- new computational methodology (algorithms and software) for large polynomials and large convex polytopes in the presence of symmetry.

\section{The SECONDARY POLYTOPE OF THE 3-CUBE}

As a warm-up for our study of the 4-cube, we first discuss our primary objects of interest for the 3-cube. Recall that the Newton polytope $\mathcal{N}(G)$ of a polynomial $G$ is the convex hull of the exponent vectors of the monomials which appear in the expansion of $G$. The Newton polytope $\mathcal{N}\left(D_{222}\right)$ of the hyperdeterminant $D_{222}$ is the convex hull in $\mathbb{R}^{8}$ of the six rows of the matrix

\begin{tabular}{|c|c|c|c|c|c|c|c|c|}
\hline & $x_{000}$ & $x_{001}$ & $x_{010}$ & $x_{011}$ & $x_{100}$ & $x_{101}$ & $x_{110}$ & $x_{111}$ \\
\hline$c_{000} c_{011} c_{101} c_{110}$ & 1 & 0 & 0 & 1 & 0 & 1 & 1 & 0 \\
\hline$c_{001} c_{010} c_{100} c_{111}$ & 0 & 1 & 1 & 0 & 1 & 0 & 0 & 1 \\
\hline$c_{000}^{2} c_{111}^{2}$ & 2 & 0 & 0 & 0 & 0 & 0 & 0 & 2 \\
\hline$c_{001}^{2} c_{110}^{2}$ & 0 & 2 & 0 & 0 & 0 & 0 & 2 & 0 \\
\hline$c_{010}^{2} c_{101}^{2}$ & 0 & 0 & 2 & 0 & 0 & 2 & 0 & 0 \\
\hline$c_{011}^{2} c_{100}^{2}$ & 0 & 0 & 0 & 2 & 2 & 0 & 0 & 0 \\
\hline
\end{tabular}

These six monomials labeling the rows are the extreme monomials of $D_{222}$, which means that their exponent vectors are vertices of the Newton polytope $\mathcal{N}\left(D_{222}\right)$. 
The other six monomials in $D_{222}$ are not extreme monomials, since their exponent vectors lie in the relative interiors of edges of the 4-dimensional polytope $\mathcal{N}\left(D_{222}\right)$. Combinatorially, $\mathcal{N}\left(D_{222}\right)$ is a bipyramid over a tetrahedron. Its $f$-vector records the number of faces of dimensions $0,1,2$, and 3 , respectively:

$$
f\left(\mathcal{N}\left(D_{222}\right)\right)=(6,14,16,8) .
$$

The Newton polytope of the $2 \times 2 \times 2$-hyperdeterminant has the following irredundant presentation by linear equations and inequalities:

$$
\begin{gathered}
\mathcal{N}\left(D_{222}\right)=\left\{\left(x_{000}, x_{001}, \ldots, x_{111}\right) \in \mathbb{R}^{8}:\right. \\
x_{000}, x_{001}, x_{010}, x_{011}, x_{100}, x_{101}, x_{110}, x_{111} \geq 0 \\
x_{000}+x_{001}+x_{010}+x_{011}=x_{000}+x_{001}+x_{100}+x_{101}=2, \\
\left.x_{000}+x_{010}+x_{100}+x_{110}=x_{001}+x_{011}+x_{101}+x_{111}=2\right\} .
\end{gathered}
$$

In Section 4, we determine the analogous presentation for $\mathcal{N}\left(D_{2222}\right)$.

We consider the principal determinant of the 3-cube. By [12, Thm. 10.B.1.2], this is the following product of determinants associated to faces and vertices:

$$
\begin{aligned}
E_{222}=D_{222} & \cdot\left(c_{000} c_{011}-c_{001} c_{010}\right) \cdot\left(c_{000} c_{101}-c_{001} c_{100}\right) \\
& \cdot\left(c_{000} c_{110}-c_{010} c_{100}\right) \cdot\left(c_{001} c_{111}-c_{011} c_{101}\right) \\
& \cdot\left(c_{010} c_{111}-c_{011} c_{110}\right) \cdot\left(c_{100} c_{111}-c_{101} c_{110}\right) \\
& \cdot c_{000} \cdot c_{001} \cdot c_{010} \cdot c_{011} \cdot c_{100} \cdot c_{101} \cdot c_{110} \cdot c_{111} .
\end{aligned}
$$

The expansion of this polynomial of degree 24 has 231 monomials, of which 74 are extreme monomials. The symmetry group of the 3 -cube, which is the Weyl group $B_{3}$ of order 48 , acts on these 231 monomials. The 74 extreme monomials come in six orbits:

$\begin{array}{cccc}\text { Type } & \text { Monomial } & \text { GKZ Vector } & \text { Orbit Size } \\ 1 & -4 c_{000} c_{001}^{5} c_{010}^{5} c_{011} c_{100}^{5} c_{101} c_{110} c_{111}^{5} & (15515115) & 2 \\ 2 & -c_{000} c_{001}^{4} c_{010}^{4} c_{011}^{3} c_{100}^{6} c_{101} c_{110} c_{111}^{4} & (14436114) & 8 \\ 3 & c_{000} c_{001}^{3} c_{010}^{4} c_{011}^{4} c_{100}^{6} c_{101}^{2} c_{110} c_{111}^{3} & (13446213) & 24 \\ 4 & c_{000} c_{001}^{3} c_{010}^{3} c_{011}^{5} c_{100}^{5} c_{101}^{3} c_{110}^{3} c_{111} & (13355331) & 12 \\ 5 & -c_{000} c_{001}^{3} c_{010}^{3} c_{011}^{5} c_{100}^{6} c_{101}^{2} c_{110}^{2} c_{111}^{2} & (13356222) & 24 \\ 6 & c_{000}^{2} c_{001}^{2} c_{010}^{2} c_{011}^{6} c_{100}^{6} c_{101}^{2} c_{110}^{2} c_{111}^{2} & (22266222) & 4\end{array}$

The Newton polytope $\mathcal{N}\left(E_{222}\right)$ of the principal determinant $E_{222}$ is the secondary polytope of the 3 -cube. It is 4 -dimensional and has the $f$-vector $(74,152,100,22)$. A Schlegel diagram of this polytope was posted by Julian Pfeifle at

\section{ww. .eg-models.de/models/Discrete_Mathematics/ Polytopes/Secondary_Polytopes/2000.09.031/.}

Using POLYMAKE [1], we found that the secondary polytope of the 3-cube has the following irredundant presentation by linear equations and inequalities:

$$
\begin{gathered}
\mathcal{N}\left(E_{222}\right)=\left\{\left(x_{000}, x_{001}, \ldots, x_{111}\right) \in \mathbb{R}^{8}:\right. \\
x_{000}+x_{001}+x_{010}+x_{011}=x_{000}+x_{001}+x_{100}+x_{101}=12, \\
x_{000}+x_{010}+x_{100}+x_{110}=x_{001}+x_{011}+x_{101}+x_{111}=12, \\
1 \leq x_{000}, x_{001}, x_{010}, x_{011}, x_{100}, x_{101}, x_{110}, x_{111} \leq 6, \\
x_{000}+x_{001} \geq 4, x_{000}+x_{010} \geq 4, x_{000}+x_{100} \geq 4, \\
\left.x_{001}+x_{011} \geq 4, x_{010}+x_{011} \geq 4, x_{001}+x_{101} \geq 4\right\}
\end{gathered}
$$


According to the theory of Gel'fand, Kapranov, and Zelevinsky [12, the 74 vertices of the secondary polytope $\mathcal{N}\left(E_{222}\right)$ are in bjiection with the regular triangulations of the 3 -cube. For the 3 -cube all triangulations are regular, so the 3 -cube has 74 triangulations which come in 6 orbits. The vertex of $\mathcal{N}\left(E_{222}\right)$ corresponding to a given triangulation $\Pi$ is called the $G K Z$ vector of $\Pi$. The $i$ th coordinate of the GKZ vector of $\Pi$ is the sum of the normalized volumes of all tetrahedra in $\Pi$ which contain the $i$ th vertex of the cube. For a general introduction to triangulations, their GKZ vectors, and secondary polytopes, see the forthcoming book by De Loera, Rambau, and Santos [10. The 6 types of triangulations of the 3-cube are depicted in [10, Figure 1.38] and [6, Figure 3]. Their tight spans, which are polyhedral complexes dual to the triangulations, are shown in Figure 1. Here is a detailed description:

Type 1. These 2 triangulations divide the cube into five tetrahedra. There is one central tetrahedron of normalized volume two and four unimodular tetrahedra which border the central tetrahedron.

The remaining five types of triangulations divide the 3-cube into six unimodular tetrahedra. Each of these triangulations uses a diagonal of the cube.

Type 2. We get these 8 triangulations by slicing off the three vertices adjacent to a fixed vertex. The remaining bipyramid is cut into three tetrahedra.

Type 3. These 24 triangulations also use a diagonal. Of the other six vertices we pick two that are diagonal on a facet, and we slice them off.

Type 4. These 12 triangulations are indexed by ordered pairs of diagonals. The end points of the first diagonal are sliced off, and the remaining octahedron is triangulated using the second diagonal.

Type 5. These 24 triangulations are indexed by a diagonal and one other vertex. That vertex is sliced off, and the remaining polytope is divided into a pentagonal ring of tetrahedra around the diagonal.

Type 6. These four triangulations are indexed by the diagonals. The cube is divided into a hexagonal ring of tetrahedra around the diagonal.

We depict each triangulation of the 3-cube by its corresponding tight span, which is the planar graph dual to the triangulation. Each vertex of the tight span corresponds to a tetrahedron in the triangulation, and two vertices are connected by an edge if the corresponding tetrahedra share a triangle. Regions of the tight span correspond to interior edges of the triangulation. The six types of tight spans are shown in Figure 1. Compare it to Figure 2 in [18.

The 22 facets of $\mathcal{N}\left(E_{222}\right)$ correspond to proper subdivisions of the 3-cube which are as coarse as possible. The eight facet inequalities like $x_{000} \geq 1$ correspond to slicing off one vertex. The eight inequalities like $x_{000} \leq 6$ correspond to subdividing the 3 -cube into three pyramids whose bases are the square facets disjoint from one vertex. The six inequalities like $x_{000}+x_{001} \geq 4$ correspond to subdividing the 3 -cube into two triangular prisms.

Since the hyperdeterminant $D_{222}$ is a factor of the principal determinant $E_{222}$, its Newton polytope $\mathcal{N}\left(D_{222}\right)$ is a Minkowski summand of the secondary polytope $\mathcal{N}\left(E_{222}\right)$. This implies the existence of a natural map from the 74 vertices of $\mathcal{N}\left(E_{222}\right)$ onto the six vertices of $\mathcal{N}\left(D_{222}\right)$. Equivalently, we have a map from the 


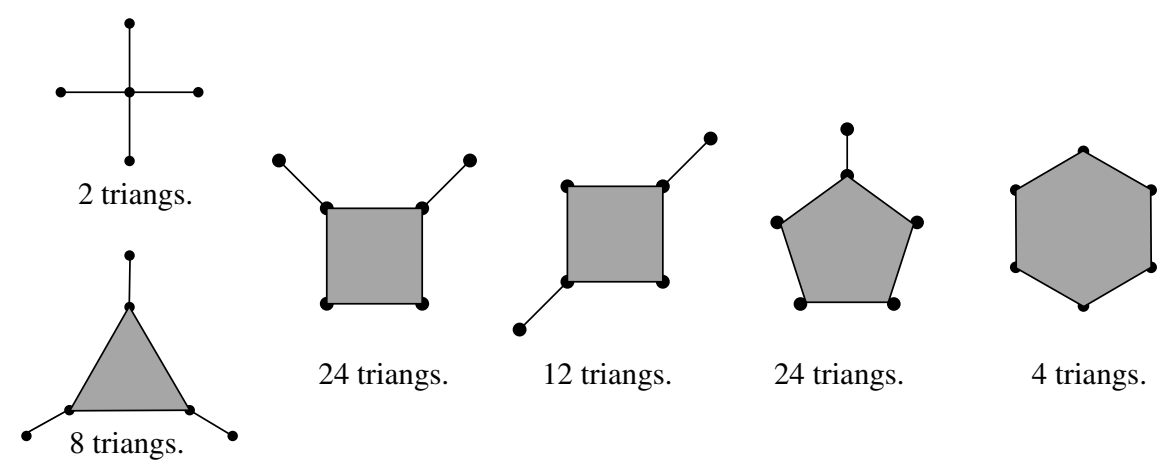

Figure 1. The tight spans dual to the six types of triangulations of the 3-cube. Each tight span represents an orbit of extreme monomials of the principal determinant $E_{222}$.

regular triangulations of the 3 -cube to the extreme monomials of $D_{222}$. A formula for this map can be derived from Theorem 3.2 in [12, §11.A].

The extreme monomials of $D_{222}$ come in two $B_{3}$-orbits. We now describe the corresponding two orbits of $D$-equivalence classes. Each class consists of all triangulations which are mapped to a fixed extreme monomial of $D_{222}$. The $D$-equivalence class of the monomial $c_{001} c_{010} c_{100} c_{111}$ consists of only one triangulation, namely the triangulation of type 1 with $\mathrm{GKZ}$ vector $(1,5,5,1,5,1,1,5)$. The $D$-equivalence class of $c_{000}^{2} c_{111}^{2}$ consists of all 18 triangulations which use the diagonal from $(0,0,0)$ to $(1,1,1)$. Thus the number of triangulations of the 3 -cube decomposes as follows into the sizes of the $D$-equivalence classes:

$$
74=2 \cdot 1+4 \cdot 18=1+1+18+18+18+18 .
$$

We emphasize that all of these results on the 3-cube are easy and well-known. This section served a purely expository purpose, namely, to set the stage for the new results on the 4 -cube to be presented in the later sections. For instance, Table 5 generalizes the identity (3) from the 3 -cube to the 4-cube.

\section{SCHLÄFLI'S FORMULA AND ITS EXPANSION}

We now consider a multilinear polynomial in four variables $x, y, z$, and $w$,

$$
\begin{aligned}
F= & c_{0000}+c_{0001} w+c_{0010} z+c_{0011} z w+c_{0100} y+c_{0101} y w \\
& +c_{0110} y z+c_{0111} y z w+c_{1000} x+c_{1001} x w+c_{1010} x z \\
& +c_{1011} x z w+c_{1100} x y+c_{1101} x y w+c_{1110} x y z+c_{1111} x y z w,
\end{aligned}
$$

where the 16 coefficients $c_{i j k l}$ are regarded as unknowns.

Slightly rephrasing the definition given in the Introduction, the hyperdeterminant $D_{2222}$ is the unique irreducible polynomial (up to sign) of content one in the 16 unknowns $c_{i j k l}$ that vanishes whenever the system of equations

$$
F=\frac{\partial F}{\partial x}=\frac{\partial F}{\partial y}=\frac{\partial F}{\partial z}=\frac{\partial F}{\partial w}=0
$$

has a solution $\left(x_{0}, y_{0}, z_{0}, w_{0}\right)$ in $\mathbb{C}^{4}$.

In theory, we can compute $D_{2222}$ by eliminating the four variables $x, y, z, w$ from the five equations (4), say by using Gröbner bases or resultants [5], but in practice 
this is infeasible. The analogous computation for a multilinear polynomial in three variables, however, is easy to do, and it yields the expression for $D_{222}$ stated in the Introduction.

Schläfli's formula for $D_{2222}$ is obtained as follows. We replace each of the eight unknowns $c_{i j k}$ in the $2 \times 2 \times 2$-hyperdeterminant $D_{222}$ by $c_{i j k 0}+c_{i j k 1} w$. The resulting expression is a polynomial of degree 4 in the variable $w$. Its coefficients are polynomials of degree 4 in the $c_{i j k l}$. The discriminant of this polynomial with respect to the variable $w$ is an expression of degree 24 in the $c_{i j k l}$. This expression is Schläfli's formula for the hyperdeterminant $D_{2222}$.

Proposition 3. The hyperdeterminant $D_{2222}$ coincides with the discriminant of $D_{222}\left(c_{i j k 0}+c_{i j k 1} w\right)$, considered as a polynomial in $w$, divided by 256 .

This proposition is a special case of [12, Theorem 14.4.1]. Its proof is based on the fact that both polynomials have degree 24 , which we know for $D_{2222}$ by 12 , Corollary 14.2.10]. For tensors of larger format (e.g., $2 \times 2 \times 2 \times 2 \times 2$ ) the same recursive method does not work so well. In general, Schläfli's formula yields the desired hyperdeterminant times a large extraneous factor.

The first assertion in Theorem 1 was proved by expanding Schläfli's formula, as follows. Using MAPLE, we expressed $D_{222}\left(c_{i j k 0}+c_{i j k 1} w\right)$ as

$$
D_{222}\left(c_{i j k 0}+c_{i j k 1} w\right)=b_{4} w^{4}+b_{3} w^{3}+b_{2} w^{2}+b_{1} w+b_{0}
$$

where each $b_{i}$ is a degree 4 polynomial in the 16 unknowns $c_{i j k l}$. These expressions for the $b_{i}$ in terms of the $c_{i j k l}$ were then substituted into the discriminant

$$
\begin{aligned}
& 256 b_{0}^{3} b_{4}^{3}-192 b_{0}^{2} b_{1} b_{3} b_{4}^{2}-128 b_{0}^{2} b_{2}^{2} b_{4}^{2}+144 b_{0}^{2} b_{2} b_{3}^{2} b_{4}-27 b_{0}^{2} b_{3}^{4} \\
& +144 b_{0} b_{1}^{2} b_{2} b_{4}^{2}-6 b_{0} b_{1}^{2} b_{3}^{2} b_{4}-80 b_{0} b_{1} b_{2}^{2} b_{3} b_{4}+18 b_{0} b_{1} b_{2} b_{3}^{3}+16 b_{0} b_{2}^{4} b_{4} \\
& -4 b_{0} b_{2}^{3} b_{3}^{2}-27 b_{1}^{4} b_{4}^{2}+18 b_{1}^{3} b_{2} b_{3} b_{4}-4 b_{1}^{3} b_{3}^{3}-4 b_{1}^{2} b_{2}^{3} b_{4}+b_{1}^{2} b_{2}^{2} b_{3}^{2} .
\end{aligned}
$$

After substituting, MAPLE was unable to expand and combine the discriminant's 16 products due to memory constraints. Instead, we used MAPLE to substitute and expand each of the 16 products (such as $b_{1}^{2} b_{2}^{2} b_{3}^{2}$ ) separately. We then wrote a $\mathrm{C}++$ program to merge the 16 expansions and divide all coefficients by 256 . The result of this merge was a lexicographically sorted list of 2894276 monomials of degree 24 in the 16 unknowns $c_{i j k l}$, each with its integer coefficient. The largest absolute value of any coefficient is 112464 .

The symmetry group of the 4 -cube is the Weyl group $B_{4}$ of order 384 . It acts on the set of monomials in $D_{2222}$ giving 9617 orbits. At our website, the 9617 lexicographically smallest monomials in each orbit are listed in lexicographic order. Each monomial is listed on a separate row, in the format

$$
\text { [[ExponentVector], Coefficient, FaceDimension, OrbitSize]. }
$$

Here FaceDimension is the dimension of the smallest face of $\mathcal{N}\left(D_{2222}\right)$ containing the exponent vector. For example, the 192 monomials in the orbit of

$$
-2 \cdot c_{0011}^{2} c_{0101}^{2} c_{0110}^{3} c_{0111}^{5} c_{1000}^{7} c_{1001} c_{1010} c_{1011} c_{1100} c_{1101}
$$

are represented by the row $[[0,0,0,2,0,2,3,5,7,1,1,1,1,1,0,0],-2,3,192]$. The distribution of the 9617 orbits according to orbit size is as follows:

$$
\begin{array}{ccccccccccc}
2 & 8 & 12 & 16 & 24 & 32 & 48 & 64 & 96 & 192 & 384 \\
2 & 6 & 6 & 16 & 27 & 24 & 142 & 90 & 577 & 2743 & 5984
\end{array}
$$


The two orbits of size two are represented by the monomial

$$
2008 \cdot c_{0001}^{3} c_{0010}^{3} c_{0100}^{3} c_{0111}^{3} c_{1000}^{3} c_{1011}^{3} c_{1101}^{3} c_{1110}^{3},
$$

whose exponent vector lies on a 3 -dimensional face, and the monomial

$$
\begin{array}{r}
112464 \cdot c_{0000} c_{0001}^{2} c_{0010}^{2} c_{0011} c_{0100}^{2} c_{0101} c_{0110} c_{0111}^{2} \\
\cdot c_{1000}^{2} c_{1001} c_{1010} c_{1011}^{2} c_{1100} c_{1101}^{2} c_{1110}^{2} c_{1111},
\end{array}
$$

which has the largest coefficient and whose exponent vector lies in the relative interior of $\mathcal{N}\left(D_{2222}\right)$. The largest odd coefficient appears in the monomial

$$
-5811 \cdot c_{0001} c_{0010} c_{0011}^{4} c_{0100}^{2} c_{0101} c_{0110}^{2} c_{0111} c_{1000}^{2} c_{1001}^{2} c_{1010}^{2} c_{1100}^{2} c_{1101}^{2} c_{1110} c_{1111}
$$

with orbit size 384, whose exponent vector lies in the relative interior of a 9-face.

The distribution of the 9617 orbits according to face dimension is as follows:

$\begin{array}{cccccccccccc}0 & 1 & 2 & 3 & 4 & 5 & 6 & 7 & 8 & 9 & 10 & 11 \\ 111 & 230 & 269 & 540 & 1145 & 1862 & 2138 & 1845 & 976 & 405 & 70 & 26\end{array}$

The computation of the above face dimensions and the computation in the rest of this section were all performed after we computed the facet representation of the Newton polytope $\mathcal{N}\left(D_{2222}\right)$. In the next section, we present all eight classes of facet inequalities and explain how we obtained them.

In the remainder of this section we discuss the 20992 monomials whose exponent vectors lie in the Newton polytope $\mathcal{N}\left(D_{2222}\right)$ but which have coefficient zero in the expansion of hyperdeterminant $D_{2222}$. They come in $69 B_{4}$-orbits. In the following table we list one representative from each orbit:

$\begin{array}{llll}0002114445101010 & 0002114454011010 & 0002114454100110 & 0002114463010110 \\ 0002115345101001 & 0002115354011001 & 0002115354100101 & 0002115363010101 \\ 0002122571111001 & 0002126126200110 & 0002136025300101 & 0002144114501100 \\ 0002216126110110 & 0002223353200002 & 0002226026200002 & 0002314235200002 \\ 0002411450141100 & 0003113444102010 & 0003114344012010 & 0003114353012001 \\ 0003114353100210 & 0003114361110300 & 0003115262010201 & 0003134114402100 \\ 0003144050220201 & 0003222350221200 & 0003223225201020 & 0003223242211101 \\ 0003225033121101 & 0003324041130201 & 0003401441132100 & 0004113371000211 \\ 0004114252100310 & 0004115153000211 & 0004115161010301 & 0004125043011111 \\ 0004215043100202 & 0005114151100410 & 0005115050110500 & 0005115060010401 \\ 0006113141102310 & 0006114060000411 & 0006212122114110 & 0011111771111100 \\ 0011113553111100 & 0011121672100011 & 0011121681010011 & 0011144151130101 \\ 0011222462020011 & 0012125136000021 & 0012142241311101 & 0012220551212001 \\ 0012310542212001 & 0012410441042010 & 0013143052100013 & 0013221333201120 \\ 0013222223212110 & 0013330251110022 & 0014220351011130 & 0014231151010131 \\ 0014320252000032 & 0014330151100023 & 0014331051010023 & 0023231152000023 \\ 0111224132130210 & 0112214132031210 & 0113213132201202 & 0114213031112202 \\ 0121212324202002 & & & \end{array}$

For instance, the underlined vector represents the monomial

$$
c_{0010} c_{0011} c_{0100} c_{0101} c_{0110} c_{0111}^{7} c_{1000}^{7} c_{1001} c_{1010} c_{1011} c_{1100} c_{1101} \text {. }
$$

The orbit of this monomial has size 96 . Each of these 69 monomials listed above does appear during the expansion of Schläfli's formula, namely, when we expand each of the 16 terms of the discriminant (5). But the coefficients of these monomials sum to zero when we merge to form $D_{2222}$. 
The distribution of the 69 orbits of "missing monomials" according to the face dimension in $\mathcal{N}\left(D_{2222}\right)$ is as follows:

$$
\begin{array}{cccccccccccc}
0 & 1 & 2 & 3 & 4 & 5 & 6 & 7 & 8 & 9 & 10 & 11 \\
0 & 0 & 15 & 3 & 20 & 13 & 7 & 5 & 6 & 0 & 0 & 0
\end{array}
$$

The following method was used to generate all lattice points in $\mathcal{N}\left(D_{2222}\right)$ and hence to find the 20992 "missing monomials" in 69 orbits. We used the five equations $A \cdot x=(24,12,12,12,12)^{T}$ and 268 facet defining inequalities of $\mathcal{N}\left(D_{2222}\right)$ which will be presented in the next section. By symmetry, it suffices to generate at least one lattice point in each $B_{4}$-orbit. We first listed all ways to assign eight nonnegative integers summing to 12 on the first facet, i.e., the first eight coordinates. There were 50388 ways in all. Per the symmetry remark above, we kept only the 1349 ways which were lex-min under $B_{3}$. We then extended each of these 1349 facet assignments to the entire 4-cube as follows. We fixed two disjoint non-parallel edges on the opposite facet, assigned all possible values for those four entries, and solved for the remaining four entries via the five linear relations above. Of the resulting 4-cube assignments, 87435 have non-negative entries. We then tested these against the facet inequalities and found that 80788 of them lay in the polytope. We now had at least one representative from each orbit of lattice points in $\mathcal{N}\left(D_{2222}\right)$. From these we found 9686 orbits of lattice points. Removing the 9617 orbits of terms which do appear in $D_{2222}$, we found precisely the 69 additional orbits listed above.

\section{Computing the Newton polytope}

In this section we present our census of the vertices, facets, and other faces of the Newton polytope of the $2 \times 2 \times 2 \times 2$-hyperdeterminant. The following result completes the proof of Theorem 1. Being the analogue of equation (11), it shows the increase in complexity when passing from the 3 -cube to the 4 -cube.

Proposition 4. The $f$-vector of the Newton polytope of $D_{2222}$ equals

$$
\begin{aligned}
f\left(\mathcal{N}\left(D_{2222}\right)\right)=\quad & (25448,178780,555280,1005946,1176976, \\
& 927244,495936,176604,39680,5012,268) .
\end{aligned}
$$

We computed the vertices of the Newton polytope $\mathcal{N}\left(D_{2222}\right)$ starting from the exponent vectors of the 2894276 monomials in 9617 orbits described in Section 3. We took advantage of the symmetry group using the following heuristics: First, we sorted the set of 9617 lex-min elements, one from each orbit, lexicographically and then chose a small lexicographically contiguous subset. Within this subset, we removed points that were redundant (i.e., not vertices) using POLYMAKE [11. Whenever a point was found to be redundant, we removed its entire $B_{4}$-orbit from our original set of points. After a few iterations we obtained the convex hull of the 9617 lex-min elements, which has 1794 vertices. The union of the orbits of those 1794 points contains 484804 points. We repeated the same process on these 484804 points, finding redundant points in a small subset and removing their whole orbits. We eventually reached a subset of 25448 points in 111 orbits which appeared to be irredundant. We verified that these points are vertices by solving 111 linear programming feasibility problems.

The tangent cone at a vertex $v$ is the set $\left\{v+w \in \mathbb{R}^{16}: v+\epsilon \cdot w \in \mathcal{N}\left(D_{2222}\right)\right.$ for some $\epsilon>0\}$. The faces of the tangent cone are in natural bijection with the faces 
of $\mathcal{N}\left(D_{2222}\right)$ containing $v$. The vertex figure of a vertex $v$ is the 10-dimensional polytope obtained by cutting off $v$ from its tangent cone.

Proof of Proposition 4 . The facets and $f$-vector of $\mathcal{N}\left(D_{2222}\right)$ were computed as follows: For each of the 111 vertex classes, we chose a representative $v$ and subtracted it from each of the other 25447 vertices. The cone generated by these 25447 difference vectors is (a translate of) the tangent cone at $v$. Using POLYMAKE, we computed the faces of all dimensions of the 111 tangent cones. These computations were fairly fast since $\mathcal{N}\left(D_{2222}\right)$ is close to being simple. We now had at least one representative from the $B_{4}$-orbit of every face of $\mathcal{N}\left(D_{2222}\right)$. Each face is represented by the set of facets containing that face. With careful relabeling, we merged the 111 lists of faces and computed their orbits, thus obtaining all faces of $\mathcal{N}\left(D_{2222}\right)$.

Here is a complete list of representatives for the 111 orbits of vertices. The upper and lower indices attached to each vector are the coefficient and the orbit size, respectively.

\begin{tabular}{|c|c|}
\hline $0002022662202000_{32}^{1} 0002022671112000_{192}^{-1}$ & $0002022680111100_{192}^{1}$ \\
\hline $0002022690010110_{64}^{-1} 0002023571022000_{384}^{1}$ & $0002023572101001_{192}^{1}$ \\
\hline $0002023580021100_{384}^{-1} 0002023581011001_{384}^{-1}$ & $0002023590010101_{192}^{1}$ \\
\hline $0002024480020200_{192}^{1} 0002026226202000_{96}^{1}$ & $0002027126112000_{384}^{-1}$ \\
\hline $0002027127101010_{192}^{1} 0002027135022000_{384}^{1}$ & $0002027136011010_{384}^{-1}$ \\
\hline $0002027145010110_{192}^{1} 0002028026111100_{192}^{1}$ & $0002028027101001_{192}^{-1}$ \\
\hline $0002028035021100_{384}^{-1} 0002028036011001_{384}^{1}$ & $0002028045010101_{192}^{-1}$ \\
\hline $0002033470032000_{192}^{-1} 0002033481100002_{96}^{1}$ & $0002033490010002_{96}^{-1}$ \\
\hline $0002036136100020_{384}^{1} 0002036145010020_{384}^{-1}$ & $0002037025122000_{192}^{1}$ \\
\hline $0002037026111010_{384}^{-1} 0002037034032000_{192}^{-1}$ & $0002037035021010_{384}^{1}$ \\
\hline $0002037036100011_{384}^{-1} 0002037045010011_{384}^{1}$ & $0002037045100002_{192}^{1}$ \\
\hline $0002037054010002_{192}^{-1} 0002046035110020_{384}^{-1}$ & $0002046044020020_{384}^{1}$ \\
\hline 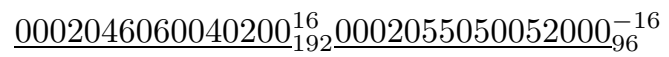 & $\underline{0002055070030002}_{96}^{-16}$ \\
\hline $0002111771112000_{96}^{1} 0002111771201100_{192}^{1}$ & $0002111780111100_{384}^{-1}$ \\
\hline $0002111781100110_{192}^{-1} 0002111790010110_{192}^{1}$ & $0002112671022000_{384}^{-1}$ \\
\hline $0002112671200200_{384}^{-1} 0002112672101001_{384}^{-1}$ & $0002112680021100_{384}^{1}$ \\
\hline $0002112680110200_{384}^{1} 0002112681011001_{192}^{1}$ & $0002112681100101_{384}^{1}$ \\
\hline $0002112690010101_{192}^{-1} 0002117117112000_{96}^{1}$ & $0002117117201100_{192}^{1}$ \\
\hline $0002117118101010_{384}^{-1} 0002117126022000_{192}^{-1}$ & $0002117126200200_{384}^{-1}$ \\
\hline $0002117127011010_{384}^{1} 0002117127100110_{384}^{1}$ & $0002117136010110_{384}^{-1}$ \\
\hline $0002118018101001_{192}^{1} 0002118027011001_{384}^{-1}$ & $0002118027100101_{384}^{-1}$ \\
\hline $0002118036010101_{384}^{1} 0002122570032000_{192}^{1}$ & $0002122581100002_{96}^{-1}$ \\
\hline $0002122590010002_{48}^{1} \mathrm{C}$ & $0002201772011010_{192}^{1}$ \\
\hline $0002201772100110_{384}^{1} 0002202662022000_{96}^{1}$ & $0002202662200200_{192}^{1}$ \\
\hline
\end{tabular}




$$
\begin{gathered}
0002202663101001_{384}^{1} 0002202672100101_{384}^{-1} \\
0002217036010002_{192}^{-1} 0002300752301100_{384}^{1} \\
0002300762200110_{192}^{-1} 0002300771110110_{192}^{1} \\
0002302563100002_{192}^{-1} 0002306136010002_{96}^{1} \\
0002400661210101_{192}^{1} 0002433050050002_{96}^{-1} \\
0003033390000003_{16}^{1} 0003035145000030_{192}^{1} \\
0003036045000021_{192}^{-1} 0003036063000003_{64}^{-1} \\
0003111671102001_{192}^{-1} 0003111680101101_{192}^{1} \\
0003117017101110_{96}^{1} 0003117026100210_{192}^{-1} \\
0004044040044000_{96}^{1} \underline{0004044080000004_{16}^{-27}} \\
0009111011107110_{48}^{1} 0009111011108001_{96}^{-1} \\
0011111772101001_{192}^{1} 0011111781011001_{192}^{-1} \\
0011120781100011_{192}^{-1} \underline{0110100990010110_{8}^{1}}
\end{gathered}
$$

$$
\begin{gathered}
0002217027100002_{96}^{1} \\
0002300761211100_{384}^{-1} \\
0002301663100011_{384}^{1} \\
0002400652300101_{384}^{-1} \\
0002500552210002_{48}^{1} \\
0003036026101020_{96}^{1} \\
0003045035100030_{192}^{1} \\
0003111690000111_{64}^{-1} \\
0003117036000111_{192}^{-1} \\
0008111111118000_{48}^{1} \\
0011110881011010_{92}^{1} \\
0011120771201001_{192}^{1} \\
0110106336010110_{32}^{1}
\end{gathered}
$$

The distribution of the 111 orbit sizes is

$$
\begin{array}{cccccccc}
8 & 16 & 32 & 48 & 64 & 96 & 192 & 384 \\
1 & 2 & 2 & 4 & 3 & 16 & 44 & 39
\end{array}
$$

The distribution of the 111 corresponding extreme monomial coefficients is

$$
\begin{array}{ccccc}
1 & -1 & 16 & -16 & -27 \\
60 & 47 & 1 & 2 & 1
\end{array}
$$

All but four of the monomials have coefficient \pm 1 . The largest absolute value of a coefficient appears in the monomial $-27 \cdot c_{0011}^{4} c_{0101}^{4} c_{0110}^{4} c_{1000}^{8} c_{1111}^{4}$ which has orbit size 16. Its $D$-equivalence class will be discussed in Example 14 The monomial $16 \cdot c_{0011}^{2} c_{0101}^{4} c_{0110}^{6} c_{1000}^{6} c_{1011}^{4} c_{1101}^{2}$ has orbit size 192, and there are two monomials with coefficient -16 , each having orbit size 96 . Their $D$-equivalence classes will be discussed in Example 15.

Among the 111 types of vertices, 35 are simple. The last underlined vertex, representing the monomial $8 \cdot c_{0001} c_{0010} c_{0100} c_{0111}^{9} c_{1000}^{9} c_{1011} c_{1101} c_{1110}$, has the largest vertex figure. That vertex figure has the $f$-vector

$$
(67,873,4405,11451,17440,16452,9699,3446,667,56) \text {. }
$$

Thus this distinguished vertex is adjacent to 67 other vertices, and it lies in 56 facets. In Example 17 we shall see that this vertex corresponds to the largest $D$ equivalence class of triangulations. Table 1 shows the distribution of the 111 types of vertices according to the number of incident edges and facets.

The 268 facets of $\mathcal{N}\left(D_{2222}\right)$ come in eight orbits. In Table 2 we present the $f$-vector and the orbit size for each facet. We discuss the geometry of each of the eight facet types. Since $\mathcal{N}\left(D_{2222}\right)$ is a Minkowski summand of the secondary polytope $\mathcal{N}\left(E_{2222}\right)$, each facet normal of $\mathcal{N}\left(D_{2222}\right)$ is a facet normal of $\mathcal{N}\left(E_{2222}\right)$ and hence corresponds to a coarsest regular subdivision of the 4-cube. We depict each subdivision by its tight span, which is the complex of bounded faces of the 
TABLE 1. Distribution of the 111 vertices according to the number of facets (columns) and edges (rows) containing the vertex.

\begin{tabular}{|c|c|c|c|c|c|c|c|c|c|c|c|c|c|c|c|c|c|c|}
\hline & 11 & 12 & 13 & 14 & 15 & 16 & 17 & 18 & 19 & 20 & 21 & 22 & 26 & 28 & 29 & 39 & 56 & total \\
\hline 11 & 35 & & & & & & & & & & & & & & & & & 35 \\
\hline 12 & & 14 & & & & & & & & & & & & & & & & 14 \\
\hline 13 & & 13 & 5 & 1 & & & & & & & & & & & & & & 19 \\
\hline 14 & & & 1 & 2 & & & & & & & & & & & & & & 3 \\
\hline 15 & & 1 & 3 & 1 & 1 & & 1 & & & & & & & & & & & 7 \\
\hline 16 & & & 1 & 1 & 2 & & & & & & & & & & & & & 4 \\
\hline 17 & & & 1 & 1 & 3 & 1 & 1 & & & & & & & & & & & 7 \\
\hline 18 & & & & & 1 & 3 & & 1 & 1 & & & & & & & & & 6 \\
\hline 19 & & & & 1 & & & 1 & & & & & & & & & & & 2 \\
\hline 20 & & & & & & & 1 & & & & & & & & & & & 1 \\
\hline 21 & & & & & & & & & 1 & 1 & 1 & & & & & & & 3 \\
\hline 22 & & & & & & & & & & & & 1 & & & & & & 1 \\
\hline 23 & & & & & 1 & & & & & & & & & & & & & 1 \\
\hline 25 & & & & & & & & & & & 1 & & 1 & & & & & 2 \\
\hline 26 & & & & & & & & & & & & & & 1 & & & & 1 \\
\hline 27 & & & & & & & & & & & & 1 & & & & & & 1 \\
\hline 30 & & & & & & & & & & & & & & 1 & & & & 1 \\
\hline 32 & & & & & & & & & & & & & & & 1 & & & 1 \\
\hline 42 & & & & & & & & & & & & & & & & 1 & & 1 \\
\hline 67 & & & & & & & & & & & & & & & & & 1 & 1 \\
\hline total & 35 & 28 & 11 & 7 & 8 & 4 & 4 & 1 & 2 & 1 & 2 & 2 & 1 & 2 & 1 & 1 & 1 & 111 \\
\hline
\end{tabular}

TABLE 2. The eight types of facets of the Newton polytope $\mathcal{N}\left(D_{2222}\right)$

\begin{tabular}{|c|c|c|}
\hline & $f$-vector & orbit \\
\hline 1 & $(11625,72614,197704,308238,303068,194347,80874,20906,3021,187)$ & 16 \\
\hline 2 & $(4112,25746,71456,115356,119228,81590,36802,10488,1704,122)$ & 12 \\
\hline 3 & $(363,2289,6538,10996,11921,8581,4080,1239,225,22)$ & 8 \\
\hline 4 & $(938,5226,13182,19716,19263,12765,5758,1721,318,31)$ & 32 \\
\hline 5 & $(336,1937,5126,8121,8468,6022,2928,950,194,22)$ & 48 \\
\hline 6 & $(289,1624,4228,6636,6894,4914,2413,798,168,20)$ & 96 \\
\hline 7 & $(450,2526,6522,10103,10315,7195,3440,1099,220,24)$ & 48 \\
\hline 8 & $(681,3906,10323,16407,17194,12264,5933,1877,357,34)$ & 8 \\
\hline
\end{tabular}

polyhedron $\left\{u \in \mathbb{R}^{5}: u \cdot A \leq w\right\}$, where $A$ is the $5 \times 16$-matrix in Section 1 and $w \in \mathbb{R}^{16}$ is the facet normal in question.

Facet 1. These 16 facets are defined by inequalities like $x_{0000} \geq 0$. The tight span of the corresponding subdivision is a line segment. The two maximal cells are the simplex obtained by slicing off one vertex and the convex hull of the other 15 vertices, which has $f$-vector $(15,34,28,9)$.

Facet 2. These 12 facets are given by inequalities like

$$
x_{0000}+x_{0001}+x_{0010}+x_{0011} \geq 2 .
$$

The tight span is a line segment. The two maximal cells in the corresponding subdivision have $f$-vector $(12,24,19,7)$. Here we cut the 4-cube with the hyperplane containing the eight vertices in a pair of opposite 2 -faces. 


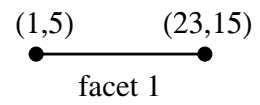

$(8,10)$

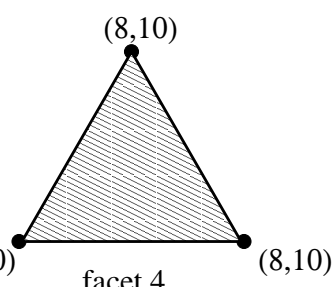

facet 4

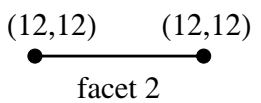

facet 2

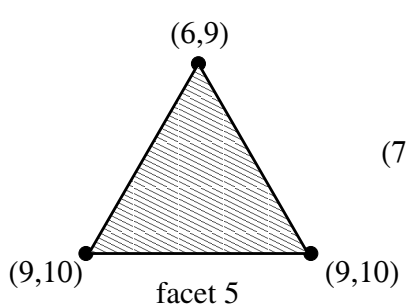

$(12,11) \quad(12,11)$

facet 3

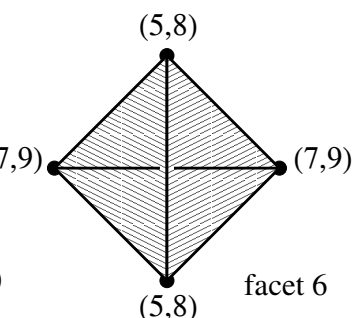

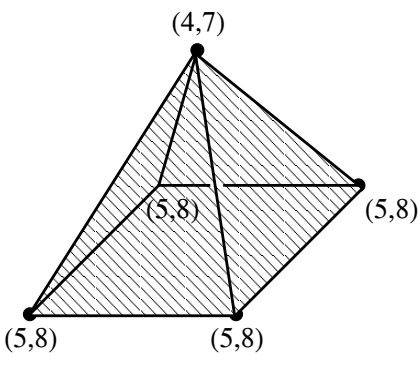

facet 7

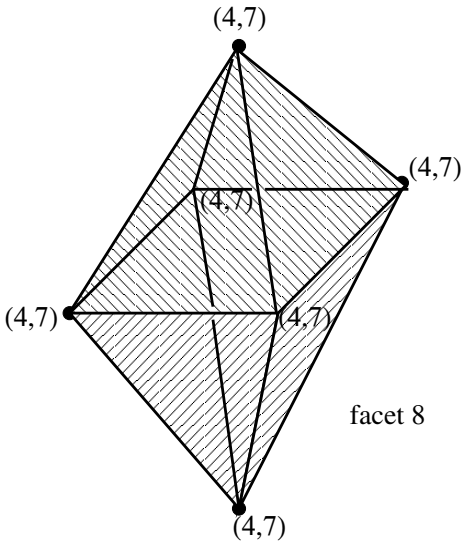

Figure 2. The tight spans dual to the subdivisions of the 4-cube corresponding to the facets of $\mathcal{N}\left(D_{2222}\right)$. Each vertex of a tight span is labeled by the normalized volume and the number of vertices of the corresponding maximal cell in the subdivision.

Facet 3. These 8 facets are given by inequalities like

$$
2 \cdot x_{0000}+x_{0001}+x_{0010}+x_{0100}+x_{1000} \geq 2 .
$$

The tight span is a line segment, and the $f$-vector of both maximal cells is $(11,28$, $26,9)$. Here we cut the 4 -cube with the hyperplane spanned by the six vertices which have coordinate sum two, whose convex hull is an octahedron.

Facet 4 . These 32 facets are given by inequalities like $x_{0000}+x_{0001} \leq 9$. The tight span is a triangle. Each of the three maximal cells is a prism over a square pyramid and has $f$-vector $(10,21,18,7)$. They are formed by fixing an edge of the 4-cube and joining that edge to the three facets which are disjoint from that edge. Any two cells intersect in a triangular prism, and all three cells intersect in the rectangle formed by the fixed edge and its opposite edge.

Facet 5. These 48 facets are given by inequalities like

$$
x_{0000}+x_{0001}+x_{0010}+x_{0100}+x_{0110}+x_{1000}+x_{1001} \geq 3 .
$$

The tight span is a triangle. Two of the three maximal cells have $f$-vector $(10,23$, $21,8)$ and intersect in a square pyramid; the other cell has $f$-vector $(9,18,15,6)$ 
and intersects each of the first two in a triangular prism. The intersection of all three cells is a square.

Facet 6 . These 96 facets are given by inequalities like

$$
x_{0000}+x_{0001}+x_{0010}+x_{0011}+x_{0100}+x_{0110}+x_{1000}+x_{1001} \geq 4 .
$$

The tight span is a tetrahedron. Two of the maximal cells have $f$-vector $(8,18,17,7)$ and the other two have $f$-vector $(9,21,20,8)$. The intersection of all four cells is a segment which is a diagonal of the 4-cube.

Facet 7. These 48 facets are given by inequalities like

$$
x_{0000}+x_{0001}+x_{0010}+x_{0011}+x_{0100}+x_{1000}+x_{1100} \leq 19 .
$$

The tight span is a square pyramid. The maximal cell corresponding to the tip of the pyramid is the convex hull of two squares in complementary dimensions that share a vertex. It has $f$-vector $(7,17,18,8)$ and meets each of the other four cells in a triangle. The other four maximal cells have $f$-vector $(8,18,17,7)$, and their intersection is a triangle. All the cells meet along a diagonal segment.

Facet 8 . These 8 facets are given by inequalities like

$$
2 \cdot x_{0000}+x_{0001}+x_{0010}+x_{0100}+x_{1000} \leq 18 .
$$

These facets are indexed by diagonals. We can identify the 4-cube with a boolean lattice when we fix a diagonal. The maximal antichain in this lattice has the structure of an octahedron. Each point in the antichain uniquely determines a maximal cell in the subdivision. Hence the tight span is also an octahedron. Each maximal cell is the convex hull of two squares in complementary dimensions joined at a vertex, and each has $f$-vector $(7,17,18,8)$.

This concludes our discussion of the facets of the Newton polytope $\mathcal{N}\left(D_{2222}\right)$. The $B_{4}$-orbits of the eight stated inequalities yield 268 facet inequalities. These together with the identity $A \cdot x=(24,12,12,12,12)^{T}$ gives an irredundant presentation of $\mathcal{N}\left(D_{2222}\right)$ by linear equations and inequalities.

\section{Tight spans and $A$-Discriminants of other 0/1-Polytopes}

The concepts and computations presented in this paper make sense for any $d \times n$ integer matrix $A$ whose row span contains the all-ones vector $(1,1, \ldots, 1)$. Following 12, the $A$-discriminant $D_{A}$ is an irreducible factor of the principal $A$-determinant $E_{A}$. The vertices of the secondary polytope $\mathcal{N}\left(E_{A}\right)$ correspond to regular triangulations of $A$, and these map onto the vertices of the Newton polytope $\mathcal{N}\left(D_{A}\right)$. The fibers of that map (i.e., the $D$-equivalence classes) and the normal fan of $\mathcal{N}\left(D_{A}\right)$ have recently been characterized in 8$]$.

For fixed $A$ and an arbitrary row vector $w \in \mathbb{R}^{n}$, we consider the polyhedron

$$
P_{w}=\left\{u \in \mathbb{R}^{d}: u \cdot A \leq w\right\} .
$$

This polyhedron is dual to the regular triangulation of $A$ defined by $w$. The tight span of $(A, w)$ is the complex of bounded faces of the polyhedron $P_{w}$. For experts in tropical geometry, we note that the $(d-2)$-skeleton of $P_{w}$ is the tropical hypersurface of $(A, w)$. The polyhedron $P_{w}$ also occurs naturally when relating regular triangulations to linear programming duality as in [10, 11.2$]$.

We propose that, for many matrices $A$ of interest in combinatorics and discrete convexity [13, the tight span is an excellent geometric representation of the data 
$(A, w)$. In Section 2 we studied the case when $A$ is the $4 \times 8$-matrix representing the 3 -cube, and in Sections 4,6 , and 7 we are concerned with the case when $A$ is the $5 \times 16$-matrix representing the 4 -cube. In what follows, we present two related situations which have appeared in the recent literature.

Example 5 (Tropical polytopes). Let $A$ be the $(r+s) \times(r \cdot s)$-matrix which represents the direct product of an $(r-1)$-simplex $\Delta_{r-1}$ with an $(s-1)$-simplex $\Delta_{s-1}$. The principal $A$-determinant $E_{A}$ is the product of all subdeterminants (of all sizes) of an $r \times s$-matrix of unknowns $\left(c_{i j}\right)$; see [12, page 303].

For instance, if $r=s=3$, then $A$ represents the product of two triangles,

$$
A=\left[\begin{array}{lllllllll}
1 & 1 & 1 & 0 & 0 & 0 & 0 & 0 & 0 \\
0 & 0 & 0 & 1 & 1 & 1 & 0 & 0 & 0 \\
0 & 0 & 0 & 0 & 0 & 0 & 1 & 1 & 1 \\
1 & 0 & 0 & 1 & 0 & 0 & 1 & 0 & 0 \\
0 & 1 & 0 & 0 & 1 & 0 & 0 & 1 & 0 \\
0 & 0 & 1 & 0 & 0 & 1 & 0 & 0 & 1
\end{array}\right]
$$

and the $A$-discriminant $D_{A}$ is the determinant of the matrix

$$
\left(\begin{array}{lll}
c_{11} & c_{12} & c_{13} \\
c_{21} & c_{22} & c_{23} \\
c_{31} & c_{32} & c_{33}
\end{array}\right) .
$$

The degree 30 polynomial $E_{A}$ is the product of all 19 subdeterminants of this matrix. The extreme monomials of $E_{A}$ appear in [12, Figure 39, page 250].

The Newton polytope $\mathcal{N}\left(E_{A}\right)$ of the product of all subdeterminants is the secondary polytope whose faces correspond to regular polyhedral subdivisions of $\Delta_{r-1}$ $\times \Delta_{s-1}$. The corresponding tight spans are precisely the tropical polytopes, which are obtained as tropical convex hulls $[9$ of $r$ points in $(s-1)$-space. The combinatorial classification of such polytopes is the analogue of what will be accomplished for the 4-cube in the next section. We refer to recent work of Santos [18 for a discussion. His Section 5 deals with tropical polytopes, and his Figure 2 is essentially the same as our Figure 1. Our number 235277 in Theorem 2 is the 4-cube analogue of the numbers in the table at the end of Section 4 in [9] including the number 35 in [6, Fig. 1] and in [9, Fig. 6].

Example 6 (Injective hulls of finite metric spaces). The term "tight span" is derived from the special case when $A$ represents the second hypersimplex. As shown in [13, 20, here the tight spans are precisely the injective hulls of finite metric spaces, which play an important role in phylogenetic combinatorics. The case studied in [20] concerns metrics on six points, where

$$
A=\left[\begin{array}{lllllllllllllll}
1 & 1 & 1 & 1 & 1 & 0 & 0 & 0 & 0 & 0 & 0 & 0 & 0 & 0 & 0 \\
1 & 0 & 0 & 0 & 0 & 1 & 1 & 1 & 1 & 0 & 0 & 0 & 0 & 0 & 0 \\
0 & 1 & 0 & 0 & 0 & 1 & 0 & 0 & 0 & 1 & 1 & 1 & 0 & 0 & 0 \\
0 & 0 & 1 & 0 & 0 & 0 & 1 & 0 & 0 & 1 & 0 & 0 & 1 & 1 & 0 \\
0 & 0 & 0 & 1 & 0 & 0 & 0 & 1 & 0 & 0 & 1 & 0 & 1 & 0 & 1 \\
0 & 0 & 0 & 0 & 1 & 0 & 0 & 0 & 1 & 0 & 0 & 1 & 0 & 1 & 1
\end{array}\right] .
$$


Here the $A$-discriminant $D_{A}$ is the determinant of the symmetric matrix

$$
\left(\begin{array}{cccccc}
0 & c_{12} & c_{13} & c_{14} & c_{15} & c_{16} \\
c_{12} & 0 & c_{23} & c_{24} & c_{25} & c_{26} \\
c_{13} & c_{23} & 0 & c_{34} & c_{35} & c_{36} \\
c_{14} & c_{24} & c_{34} & 0 & c_{45} & c_{46} \\
c_{15} & c_{25} & c_{35} & c_{45} & 0 & c_{56} \\
c_{16} & c_{26} & c_{36} & c_{46} & c_{56} & 0
\end{array}\right) .
$$

Up to a constant, the principal $A$-determinant $E_{A}$ is the product of all principal minors of size $\geq 3$. This is a polynomial of degree 156 having 194160 extreme terms in 339 symmetry classes [20, Theorem 1]. Thus our number 235277 is the 4 -cube analogue to the number 339 of generic six-point metrics.

The prominent role of the second hypersimplex in phylogenetic combinatorics raises the question of what happens for other hypersimplices. We propose the following problem for further mathematical and computational research.

Problem 7 (The hypersimplex $\Delta(6,3)$ ). Let $A$ be the $6 \times 20$-matrix whose columns are the vertices $e_{i}+e_{j}+e_{k}(1 \leq i<j<k \leq 6)$ of the third hypersimplex $\Delta(6,3)$. What is the degree of the $A$-discriminant $D_{A}$ ? Can the 14-dimensional polytope $\mathcal{N}\left(D_{A}\right)$ be computed? Can the monomial expansion of the $A$-discriminant $D_{A}$ be computed?

All matrices $A$ in the examples above have their entries in $\{0,1\}$, so they represent subpolytopes of cubes of appropriate dimensions. In what follows, we examine the $A$-discriminants of various subpolytopes of the 4 -cube. These arise naturally as irreducible factors in the initial forms of the hyperdeterminant.

For any vector $w$ in $\mathbb{R}^{16}$ we define the initial form $\operatorname{in}_{w}\left(D_{2222}\right)$ as the sum of all terms in $D_{2222}$ having minimal $w$-weight. If $w$ is generic, then $\operatorname{in}_{w}\left(D_{2222}\right)$ is a monomial, and we have classified all of these monomials in Section 4 . We now consider the other extreme case when $w$ is as non-generic as possible. More precisely, we pick $w$ among the normal vectors to the facets of $\mathcal{N}\left(D_{2222}\right)$.

Proposition 8 (All maximal initial forms of the hyperdeterminant). The following list is the classification of the initial forms of hyperdeterminant $D_{2222}$ corresponding to all facets of its Newton polytope. The eight symmetry classes of facets of $\mathcal{N}\left(D_{2222}\right)$ are listed in the same order as in Section 4.

Facet 1. If $w=e_{0000}$, then $\operatorname{in}_{w}\left(D_{2222}\right)$ is obtained from $D_{2222}$ by setting $c_{0000}=0$. This initial form is irreducible: It is the $A$-discriminant where $A$ is the 15 -point configuration obtained from the 4-cube by removing one vertex.

Facet 2. If $w=e_{0000}+e_{0001}+e_{0010}+e_{0011}$, then $\operatorname{in}_{w}\left(D_{2222}\right)$ has 67230 terms and factors as $c_{1010} c_{1001}-c_{1000} c_{1011}$ times $c_{0101} c_{0110}-c_{0100} c_{0111}$ times the square of the $2 \times 2 \times 2$-hyperdeterminant of $\left[c_{1000}, c_{1001}, c_{1010}, c_{1011}, c_{0100}, c_{0101}, c_{0110}, c_{0111}\right]$ times the product of two larger factors, each having 66 terms of degree six, which are the $A$-discriminants of the two maximal cells in the subdivision. 
Facet 3. If $w=2 \cdot e_{0000}+e_{0001}+e_{0010}+e_{0100}+e_{1000}$, then $\operatorname{in}_{w}\left(D_{2222}\right)$ equals

$$
\begin{array}{r}
(1 / 4) \cdot c_{0011} c_{0101} c_{0110} c_{1001} c_{1010} c_{1100} \cdot\left\{\operatorname{det}\left[\begin{array}{ccccc}
0 & c_{0011} & c_{0101} & c_{1001} \\
c_{0011} & 0 & c_{0110} & c_{1010} \\
c_{0101} & c_{0110} & 0 & c_{1100} \\
c_{1001} & c_{1010} & c_{1100} & 0
\end{array}\right]\right\}^{2} \\
\times \operatorname{det}\left[\begin{array}{cccccc}
2 c_{0000} & c_{0001} & c_{0010} & c_{0100} & c_{1000} \\
c_{0001} & 0 & c_{0011} & c_{0101} & c_{1001} \\
c_{0010} & c_{0011} & 0 & c_{0110} & c_{1010} \\
c_{0100} & c_{0101} & c_{0110} & 0 & c_{1100} \\
c_{1000} & c_{1001} & c_{1010} & c_{1100} & 0
\end{array}\right] \operatorname{det}\left[\begin{array}{ccccc}
2 c_{1111} & c_{1110} & c_{1101} & c_{1011} & c_{0111} \\
c_{1110} & 0 & c_{1100} & c_{1010} & c_{0110} \\
c_{1101} & c_{1100} & 0 & c_{1001} & c_{0101} \\
c_{1011} & c_{1010} & c_{1001} & 0 & c_{0011} \\
c_{0111} & c_{0110} & c_{0101} & c_{0011} & 0
\end{array}\right] .
\end{array}
$$

The squared factor is the $A$-discriminant of the octahedron, and the $5 \times 5$-determinants are $A$-discriminants of the two maximal cells in this subdivision.

Facet 4. If $w=-e_{0000}-e_{0001}$, then $\operatorname{in}_{w}\left(D_{2222}\right)$ equals

$$
\begin{gathered}
\left(c_{0000} c_{1111}-c_{0001} c_{1110}\right)^{3} \\
\cdot\left(c_{1100} c_{1111}-c_{1101} c_{1110}\right)\left(c_{1010} c_{1111}-c_{1011} c_{1110}\right)\left(c_{0110} c_{1111}-c_{0111} c_{1110}\right) \\
\cdot\left(c_{0000}^{2} c_{1001} c_{1111}-c_{0000}^{2} c_{1011} c_{1101}-c_{0000} c_{0001} c_{1000} c_{1111}-c_{0000} c_{0001} c_{1001} c_{1110}\right. \\
\left.+c_{0000} c_{0001} c_{1010} c_{1101}+c_{0000} c_{0001} c_{1011} c_{1100}+c_{0001}^{2} c_{1000} c_{1110}-c_{0001}^{2} c_{1010} c_{1100}\right) \\
\cdot\left(c_{0000}^{2} c_{0101} c_{1111}-c_{0000}^{2} c_{0111} c_{1101}-c_{0000} c_{0001} c_{0100} c_{1111}-c_{0000} c_{0001} c_{0101} c_{1110}\right. \\
\left.+c_{0000} c_{0001} c_{0110} c_{1101}+c_{0000} c_{0001} c_{0111} c_{1100}+c_{0001}^{2} c_{0100} c_{1110}-c_{0001}^{2} c_{0110} c_{1100}\right) \\
\cdot\left(c_{0000}^{2} c_{0011} c_{1111}-c_{0000}^{2} c_{0111} c_{1011}-c_{0000} c_{0001} c_{0010} c_{1111}-c_{0000} c_{0001} c_{0011} c_{1110}\right. \\
\left.+c_{0000} c_{0001} c_{0110} c_{1011}+c_{0000} c_{0001} c_{0111} c_{1010}+c_{0001}^{2} c_{0010} c_{1110}-c_{0001}^{2} c_{0110} c_{1010}\right) .
\end{gathered}
$$

The quartic factors are the $A$-discriminants of the three maximal cells.

Facet 5. If $w=e_{0000}+e_{0001}+e_{0010}+e_{0100}+e_{0110}+e_{1000}+e_{1001}$, then $\operatorname{in}_{w}\left(D_{2222}\right)$ equals $c_{0000} c_{0011} c_{0101} c_{1010} c_{1100}$ times $\left(c_{0011} c_{1100}-c_{0101} c_{1010}\right)^{3}$ times

$$
\begin{aligned}
& c_{0011} c_{1100} c_{1111}-c_{0011} c_{1101} c_{1110}-c_{0101} c_{1010} c_{1111} \\
& +c_{0101} c_{1011} c_{1110}+c_{0111} c_{1010} c_{1101}-c_{0111} c_{1011} c_{1100}
\end{aligned}
$$

times the product of two larger factors, each having 15 terms of degree five which are the $A$-discriminants of the two cells with $f$-vector $(10,23,21,8)$.

In the remaining cases the tight spans are three-dimensional (Figure 2), and the initial form $\operatorname{in}_{w}\left(D_{2222}\right)$ factors as a monomial times the product of the $A$ discriminants of the maximal cells in that subdivision of the 4-cube.

Facet 6. If $w=e_{0000}+e_{0001}+e_{0010}+e_{0011}+e_{0100}+e_{0110}+e_{1000}+e_{1001}$, then $\operatorname{in}_{w}\left(D_{2222}\right)$ has 404 terms and factors as a monomial times the product of the $A$-discriminants of the four maximal cells:

$$
\begin{gathered}
\operatorname{in}_{w}\left(D_{2222}\right)=c_{0000} \cdot c_{1100} \cdot c_{1010}^{4} \cdot c_{0101}^{4} \\
\cdot\left(c_{0101} c_{1010} c_{1111}-c_{0101} c_{1011} c_{1110}-c_{0111} c_{1010} c_{1101}+c_{0111} c_{1011} c_{1100}\right) \\
\cdot\left(c_{0000} c_{0111} c_{1011}-c_{0001} c_{0111} c_{1010}-c_{0010} c_{0101} c_{1011}+c_{0011} c_{0101} c_{1010}\right) \\
\quad \cdot\left(c_{0000} c_{0101} c_{0111} c_{1110}-c_{0000} c_{0111}^{2} c_{1100}-c_{0010} c_{0101}^{2} c_{1110}\right. \\
\left.\quad+c_{0010} c_{0101} c_{0111} c_{1100}-c_{0100} c_{0101} c_{0111} c_{1010}+c_{0101}^{2} c_{0110} c_{1010}\right) \\
\quad\left(c_{0000} c_{1010} c_{1011} c_{1101}-c_{0000} c_{1011}^{2} c_{1100}-c_{0001} c_{1010}^{2} c_{1101}\right. \\
\left.\quad+c_{0001} c_{1010} c_{1011} c_{1100}-c_{0101} c_{1000} c_{1010} c_{1011}+c_{0101} c_{1001} c_{1010}^{2}\right)
\end{gathered}
$$


Facet 7. If $w=-e_{0000}-e_{0001}-e_{0010}-e_{0011}-e_{0100}-e_{1000}-e_{1100}$, then

$$
\begin{gathered}
\operatorname{in}_{w}\left(D_{2222}\right)=c_{1111} \cdot c_{0011}^{4} \cdot c_{1100}^{4} \\
\cdot\left(c_{0000} c_{0011} c_{1100}-c_{0001} c_{0010} c_{1100}-c_{0011} c_{0100} c_{1000}\right) \\
\cdot\left(c_{0010} c_{1000} c_{1111}-c_{0010} c_{1011} c_{1100}-c_{0011} c_{1000} c_{1110}+c_{0011} c_{1010} c_{1100}\right) \\
\cdot\left(c_{0010} c_{0100} c_{1111}-c_{0010} c_{0111} c_{1100}-c_{0011} c_{0100} c_{1110}+c_{0011} c_{0110} c_{1100}\right) \\
\cdot\left(c_{0001} c_{1000} c_{1111}-c_{0001} c_{1011} c_{1100}-c_{0011} c_{1000} c_{1101}+c_{0011} c_{1001} c_{1100}\right) \\
\cdot\left(c_{0001} c_{0100} c_{1111}-c_{0001} c_{0111} c_{1100}-c_{0011} c_{0100} c_{1101}+c_{0011} c_{0101} c_{1100}\right) .
\end{gathered}
$$

Facet 8. If $w=-2 \cdot e_{0000}-e_{0001}-e_{0010}-e_{0100}-e_{1000}$, then $\operatorname{in}_{w}\left(D_{2222}\right)$ equals

$$
\begin{aligned}
c_{0000}^{3} \cdot c_{1111}^{3} \cdot & \left(c_{0000} c_{1100} c_{1111}-c_{0000} c_{1101} c_{1110}-c_{0100} c_{1000} c_{1111}\right) \\
& \cdot\left(c_{0000} c_{1010} c_{1111}-c_{0000} c_{1011} c_{1110}-c_{0010} c_{1000} c_{1111}\right) \\
& \cdot\left(c_{0000} c_{0110} c_{1111}-c_{0000} c_{0111} c_{1110}-c_{0010} c_{0100} c_{1111}\right) \\
& \cdot\left(c_{0000} c_{1001} c_{1111}-c_{0000} c_{1011} c_{1101}-c_{0001} c_{1000} c_{1111}\right) \\
& \cdot\left(c_{0000} c_{0101} c_{1111}-c_{0000} c_{0111} c_{1101}-c_{0001} c_{0100} c_{1111}\right) \\
& \cdot\left(c_{0000} c_{0011} c_{1111}-c_{0000} c_{0111} c_{1011}-c_{0001} c_{0010} c_{1111}\right) .
\end{aligned}
$$

These formulas show that $A$-discriminants for subconfigurations of the cube appear naturally as irreducible factors of leading forms of the hyperdeterminant. This suggests the general problem of studying $A$-discriminants and the related tight spans for various families of 0/1-polytopes. Other natural classes of configurations for which a study of $A$-discriminants would be interesting include generalized permutohedra, Birkhoff polytopes, and reflexive polytopes.

\section{VERTICES OF THE SECONDARY POLYTOPE}

The 4 -cube is given by the $5 \times 16$-matrix $A$ in the Introduction. For any generic vector $w \in \mathbb{R}^{16}$ we consider the tight span of $(A, w)$ whose cells are the bounded faces of the simple polyhedron $P_{w}=\left\{u \in \mathbb{R}^{5}: u \cdot A \leq w\right\}$. Each vertex of $P_{w}$ is indexed by five columns of $A$. This collection of 5 -tuples, each regarded as a 4-simplex, is a regular triangulation $\Pi_{w}$ of the 4-cube (cf. [10, §5]).

The regular triangulations of the 4-cube are in bijection with the vertices of the secondary polytope. As in Section 2, we define the secondary polytope as the Newton polytope $\mathcal{N}\left(E_{2222}\right)$ of the principal determinant $E_{2222}$, which is the following product of determinants associated to all faces of the 4-cube:

- 16 linear factors $c_{i j k l}$ corresponding to the vertices,

- 24 quadratic factors $D_{22}$ corresponding to the 2-faces,

- 8 quartic factors $D_{222}$ corresponding to the facets,

- the hyperdeterminant $D_{2222}$ corresponding to the solid 4-cube.

Thus the principal determinant $E_{2222}$ is a polynomial in the 16 unknowns $c_{i j k l}$ of degree $120=16 \cdot 1+24 \cdot 2+8 \cdot 4+1 \cdot 24$. The vertices of its Newton polytope $\mathcal{N}\left(E_{2222}\right)$ are the $\mathrm{GKZ}$ vectors of the regular triangulations of the 4-cube.

Recall from the first part of Theorem 2 that the 4-cube has 87959448 regular triangulations, or, equivalently, $\mathcal{N}\left(E_{2222}\right)$ has 87959448 vertices. Up to the action of the Weyl group $B_{4}$, there are 235277 types of regular triangulations. Thus, this is the number of combinatorially distinct simple polyhedra $P_{w}$ and also the number of generic tight spans. 

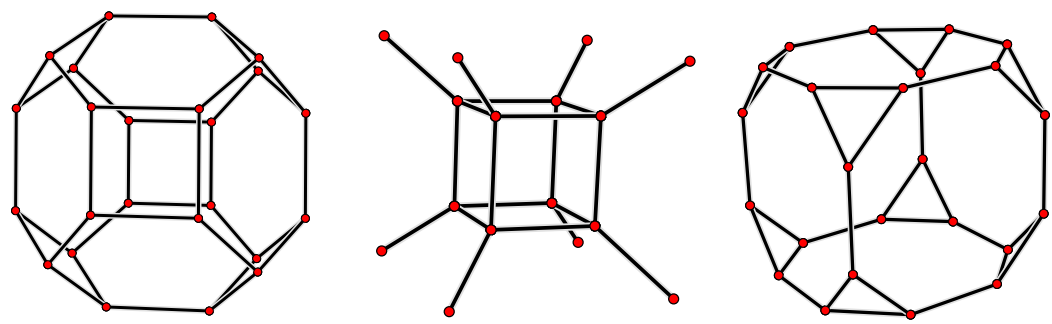

FiguRE 3. Tight spans of the three triangulations in Example 10.

The main objective of this section is to provide a detailed study of the regular triangulations of the 4-cube, with emphasis on their tight spans. We present a range of results which were computed from our data.

Corollary 9. The distribution of the 235277 symmetry classes of vertices of the secondary polytope $\mathcal{N}\left(E_{2222}\right)$ according to orbit size is as follows:

$\begin{array}{ccccccccc}8 & 16 & 24 & 32 & 48 & 64 & 96 & 192 & 384 \\ \underline{3} & 7 & 2 & 13 & 48 & 102 & 516 & 11357 & 223229\end{array}$

Example 10 (The most symmetric triangulations). One of the triangulations with orbit size 8 is the staircase triangulation. Its tight span is a solid permutohedron, and its GKZ vector is $(4,6,6,24,6,4,4,6,6,4,4,6,24,6,6,4)$. Another is the unique triangulation that uses 16 maximal simplices. Eight simplices have volume one and eight have volume two. Its GKZ vector is $(1,12,12,1,12,1,1,20,20,1,1$, $12,1,12,12,1)$, and its tight span is a 3 -cube with tentacles attached to its vertices. The third triangulation with orbit size 8 has a solid truncated 3 -cube as its tight span. Its GKZ vector is $(3,8,8,3,8,3,3,24,24,3,3,8,3,8,8,3)$. The tight spans of these symmetric triangulations are shown in Figure 3 .

Table 3 classifies the regular triangulations according to the number of maximal simplices used. It is well-known that the minimal number is sixteen (cf. [3]). We see that any triangulation can only use at most one simplex of volume three, since all such simplices contain the centroid of the cube as an interior point. There are 16 simplices of volume three, all of which are $B_{4}$-equivalent; a representative is $\{(1000),(1111),(0011),(0101),(0110)\}$.

Example 11 (Triangulating the 4-cube with 17 simplices). Table 3 shows that there is a unique regular triangulation with 17 maximal simplices, ten of volume one and seven of volume two. Its tight span, shown in Figure 4, is a cube with a truncated vertex and tentacles attached to the seven original vertices. Its GKZ vector is $(1,11,12,1,12,1,1,21,20,3,1,11,1,11,12,1)$.

Table 4 classifies the tight spans according to their $f$-vectors and their signature, by which we mean the set of dimensions of the maximal cells. Note that every triangulation of the 4-cube has at most one interior edge (namely, a diagonal), so each tight span has at most one 3 -dimensional cell. The $f$-vector of a tight span is determined by the number of vertices and 3-cells, i.e., the number of maximal simplices and diagonals used in the triangulation. 
TABLE 3. Classification of regular triangulations of the 4-cube according to the number and volumes of maximal simplices

\begin{tabular}{|c|c|c|c|c|}
\hline $\begin{array}{l}\text { \# maximal } \\
\text { simplices }\end{array}$ & volume 1 & \# simplices of & volume 3 & $\begin{array}{l}\# B_{4} \text {-orbits of } \\
\text { triangulations }\end{array}$ \\
\hline 16 & 8 & 8 & $\overline{0}$ & 1 \\
\hline 17 & 10 & 7 & $\overline{0}$ & $\overline{1}$ \\
\hline 18 & 12 & 6 & $\overline{0}$ & 6 \\
\hline 18 & 13 & 4 & 1 & 11 \\
\hline 19 & 14 & 5 & 0 & 25 \\
\hline 19 & 15 & 3 & 1 & 48 \\
\hline 20 & 16 & 4 & 0 & 628 \\
\hline 20 & 17 & 2 & 1 & 344 \\
\hline 21 & 18 & 3 & 0 & 5847 \\
\hline 21 & 19 & 1 & 1 & 1263 \\
\hline 22 & 20 & 2 & 0 & 24499 \\
\hline 22 & 21 & 0 & 1 & 1967 \\
\hline 23 & 22 & 1 & 0 & 48648 \\
\hline 24 & 24 & $\overline{0}$ & $\overline{0}$ & 151989 \\
\hline
\end{tabular}

TABLE 4. Distribution of the 235277 types of triangulations. The rows correspond to the $f$-vectors and the columns correspond to the dimensions of maximal faces of the tight span.

\begin{tabular}{|c|c|c|c|c|c|c|c|}
\hline & $\{3\}$ & $\{3,2\}$ & $\{3,1\}$ & $\{3,2,1\}$ & $\{2\}$ & $\{2,1\}$ & total \\
\hline$(16,20,6,1)$ & & & 1 & & & & 1 \\
\hline$(17,22,7,1)$ & & & 1 & & & & 1 \\
\hline$(18,24,8,1)$ & & & 4 & 2 & & & 6 \\
$(18,23,6,0)$ & & & & & & 11 & 11 \\
\hline$(19,26,9,1)$ & & & 6 & 19 & & & 25 \\
$(19,25,7,0)$ & & & & & & 48 & 48 \\
\hline$(20,28,10,1)$ & & 1 & 23 & 209 & & & 233 \\
$(20,27,8,0)$ & & & & & 24 & 715 & 739 \\
\hline$(21,30,11,1)$ & & 5 & 34 & 1372 & & & 1411 \\
$(21,29,9,0)$ & & & & & 392 & 5307 & 5699 \\
\hline$(22,32,12,1)$ & & 112 & 84 & 9342 & & & 9538 \\
$(22,31,10,0)$ & & & & & 2156 & 14772 & 16928 \\
\hline$(23,34,13,1)$ & & 2116 & 100 & 46432 & & & 48648 \\
\hline$(24,36,14,1)$ & 125 & 27054 & & 124810 & & & 151989 \\
\hline total & 125 & 29288 & 253 & 182186 & 2572 & 20853 & 235277 \\
\hline
\end{tabular}

Example 12 (The smallest tight spans of signature $\{3,2\}$ and $\{3,2,1\}$ ). The unique tight span of signature $\{3,2\}$ with 20 vertices contains a solid tetrahedron with six hexagons attached, one to each of the tetrahedron's edges. See Figure 4. Its triangulation has GKZ vector $(5,2,2,15,2,15,15,4,24,5,5,2,5,2,2,15)$ and lies in the 88 th $D$-equivalence class.

There are two tight spans of signature $\{3,2,1\}$ with 18 vertices. Their GKZ vectors are $(1,9,13,2,12,1,1,21,19,6,1,9,1,11,12,1)$ and $(1,12,12,1,11,1,1,21,19$, 

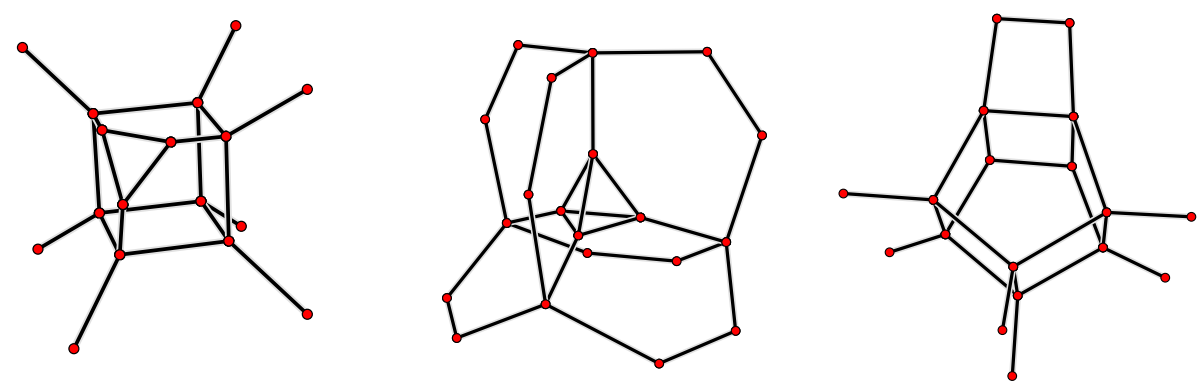

Figure 4. The triangulations in Examples 11 and 12

$1,1,13,5,10,10,1)$. They are both in the largest $D$-equivalence class. One of them is shown in Figure 4. The other has a triangle and a tentacle in place of the rectangular maximal 2 -face.

The first column in Table 4 is especially interesting as it ties in with an active area of research in combinatorial commutative algebra. Using the language introduced in [4, the $d$-cube is a Gorenstein polytope, and the tight spans of signature $\{d-1\}$ are precisely its Gorenstein triangulations. The 3-cube has only one Gorenstein triangulation, corresponding to the last type in Figure 1

Example 13 (The Gorenstein triangulations of the 4-cube). The 4-cube has precisely 125 types of Gorenstein triangulations. Two of them are pictured in Figure 3. The Stanley-Reisner ideal of a Gorenstein triangulation corresponds to an initial ideal of the Segre variety $\mathbb{P}^{1} \times \mathbb{P}^{1} \times \mathbb{P}^{1} \times \mathbb{P}^{1} \subset \mathbb{P}^{15}$ that is squarefree and Gorenstein. The minimal free resolution of the Alexander dual of this monomial ideal is cellular and is supported on the tight span itself. For tropical polytopes (Example 5) this was shown in [2], but the same holds for unimodular regular triangulations of arbitrary polytopes, including the 4-cube. All 125 Gorenstein tight spans are deformed permutohedra, and it would be interesting to study their combinatorics in arbitrary dimensions $d \geq 4$.

We now return to our discussion of the hyperdeterminant $D_{2222}$. Since $D_{2222}$ is a factor of the principal determinant $E_{2222}$, there is a natural many-to-one map from the vertices of $\mathcal{N}\left(E_{2222}\right)$ to the vertices of $\mathcal{N}\left(D_{2222}\right)$. Two regular triangulations are $D$-equivalent if they are mapped to the same vertex of $\mathcal{N}\left(D_{2222}\right)$. For the 3 -cube the $D$-equivalence classes were described in Section 2 . Table 5 shows the decomposition of the number 87959448 of regular triangulations of the 4-cube into the cardinalities of the orbits of $D$-equivalence classes. Thus the identity expressed in Table 5 is the 4-cube version of the identity (3) for the 3-cube. The 111 orbits of $D$-equivalence classes are listed in the order of the corresponding orbits of vertices of $\mathcal{N}\left(D_{2222}\right)$ in Section 4.

In the triangulations of the 4 -cube, the centroid $\left(1, \frac{1}{2}, \frac{1}{2}, \frac{1}{2}, \frac{1}{2}\right)$ is in the relative interior of one of the following three kinds of simplices: a 4-simplex of normalized volume 3 , a tetrahedron of normalized volume 2 , or a diagonal.

Example 14 (The "coefficient -27 class"). The triangulations which use a fixed simplex of normalized volume three form a single $D$-equivalence class of cardinality 82832. This class corresponds to the vertex $0004044080000004_{16}^{-27}$ of $\mathcal{N}\left(D_{2222}\right)$. The 
TABLE 5. Decomposition into $D$-equivalence classes

\begin{tabular}{|c|c|c|c|c|c|c|c|c|c|c|}
\hline \multicolumn{11}{|l|}{87959448} \\
\hline 32 & 2116 & + & 192 & 644 & + & 192 & 2752 & + & 64 & 03 \\
\hline 384 & 155 & + & 92 & & + & 38 & & + & 384 & \\
\hline 192 & 10384 & + & 19 & 52 & + & 96 & & + & 384 & \\
\hline 192 & 140 & + & 38 & & + & 38 & & 1 & 15 & \\
\hline 192 & 2584 & + & 192 & & + & 384 & 4122 & + & 38 & \\
\hline 192 & 9090 & + & 19 & & + & 96 & & 1 & & \\
\hline 384 & 318 & + & 38 & 58 & + & 192 & & 1 & $3 \varepsilon$ & \\
\hline 192 & 514 & + & 38 & & + & & & 1 & 38 & \\
\hline 192 & 6 & + & 19 & & + & 384 & & + & 38 & \\
\hline 192 & $\underline{18964}$ & + & 9 & & + & 96 & 2 & & & \\
\hline 192 & & + & 384 & & + & 19 & & t & 19 & 180 \\
\hline 384 & 45 & + & 384 & & + & & 2 & + & & \\
\hline 384 & $7 \varepsilon$ & + & 19 & & + & 384 & & 1 & 19 & \\
\hline 96 & & + & 19 & & + & 34 & 1360 & + & & \\
\hline 384 & & + & 384 & & + & 84 & 0 & + & 38 & \\
\hline 192 & & + & 384 & 52 & + & 384 & 0 & + & 384 & \\
\hline 92 & & + & 96 & & + & 4 & & + & & \\
\hline 192 & 4056 & + & 384 & 92 & + & 96 & 2 & + & 192 & \\
\hline 384 & & + & 3 & & + & 96 & 0 & + & 192 & 16 \\
\hline 384 & 1096 & + & 38 & & + & 192 & 8 & + & 192 & 18 \\
\hline 384 & & + & 192 & & + & 9 & & + & 384 & \\
\hline 192 & & + & 9 & 84 & + & 48 & 52 & 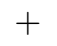 & 16 & 18 \\
\hline 192 & 4600 & + & 0 & 2888 & + & 19 & 2700 & + & 64 & 235 \\
\hline 192 & 514 & r & 19 & & + & 19 & 424 & & 64 & \\
\hline 96 & 400 & + & 192 & 200 & + & 192 & 580 & r & 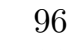 & 83 \\
\hline 16 & $\underline{82832}$ & + & 48 & 1764 & + & 48 & 6200 & + & 0 & 581 \\
\hline 19 & 4860 & + & 192 & 96 & + & 192 & 288 & + & 192 & \\
\hline 152 & 1240 & & & $\underline{349555}$ & & 32 & $\underline{04}$ & & & \\
\hline
\end{tabular}

corresponding monomial in $D_{2222}$ has the largest absolute value of any coefficient, namely -27 , among all the extreme monomials.

Example 15 (The "coefficient \pm 16 classes"). The triangulations in which the centroid is in a fixed tetrahedron of volume two form a $D$-equivalence class corresponding to one of the extreme monomials of $D_{2222}$ with coefficient \pm 16 , which come in three symmetry classes underlined in Table 5 and following the proof of Proposition 4 . There are 24 such tetrahedra, all lying in a single $B_{4}$ orbit. The hyperplane spanned by the special tetrahedron contains eight vertices of the 4-cube, and there are four vertices on each side. In each triangulation, one vertex from each side is joined to the special tetrahedron. The Hamming distance between these two vertices can be two, three, or four, and they correspond precisely to the three $D$ equivalence classes with coefficient \pm 16 . The first underlined class corresponds to Hamming distance three where there are two choices on one side after the vertex on the other side is chosen. Hence it has orbit size $24 \times 4 \times 2=192$. The second and third underlined classes correspond to Hamming distances four and two, respectively, and have orbit size $24 \times 4=96$. 

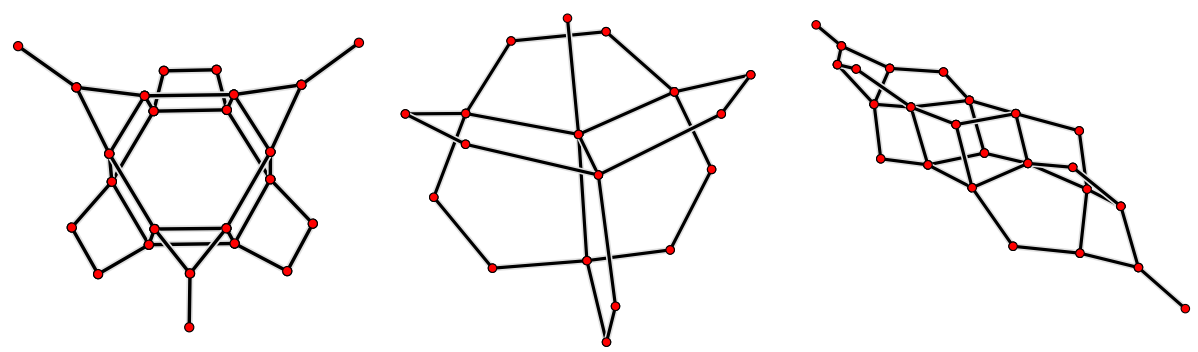

Figure 5. Tight spans of triangulations in Examples 16, 14, and 18

The other $D$-equivalence classes contain only triangulations using a diagonal of the 4-cube, and all those classes contain some unimodular triangulations.

Example 16 (The smallest $D$-equivalence class). This class has only 64 triangulations and corresponds to the last summand in Table 5. The tight span of each of these 64 triangulations contains a hexagonal prism and six lower-dimensional pieces attached to alternating edges of the prism, as shown on the left in Figure 5. Each of these six lower-dimensional pieces is either a rectangle or a triangle with a tentacle, whence the number $2^{6}=64$.

Example 17 (The largest $D$-equivalence class). This class has 349555 triangulations and corresponds to the vertex of $\mathcal{N}\left(D_{2222}\right)$ which has the largest vertex figure and the smallest orbit size. It contains all the triangulations in Examples 10, 11, and 13

Using our data, it is easy to study other questions concerning regular triangulations of the 4-cube. Answering a question which was left open in [19], we found that the 4-cube does not possess any delightful triangulations. Such a triangulation would correspond to a square-free initial ideal of the first secant variety of $\mathbb{P}^{1} \times \mathbb{P}^{1} \times \mathbb{P}^{1} \times \mathbb{P}^{1} \subset \mathbb{P}^{15}$, which has dimension 9 and degree 64 . For every regular triangulation $\Pi$ we computed the number of 10 -element subsets of the vertices of the 4-cube which are the union of vertices of two simplices in $\Pi$. That number is bounded above by 64 , and $\Pi$ would be delightful if equality holds.

Example 18 (The most delightful triangulation). We found that the maximum number of 10-sets of vertices which are the unions of vertices of two simplices in a regular triangulation is 56 . This number is attained by two triangulations which are both in $D$-equivalence classes of type 11 . Their GKZ vectors are $(1,4,4,11,6,13,9$, $12,12,9,13,6,11,4,4,1)$ and $(1,4,4,11,6,13,9,12,12,11,13,4,9,4,6,1)$. Both of the corresponding tight spans have $f$-vector $(24,36,14,1)$ and consist of two maximal edges, eight maximal 2-faces, and one 3 -cube. The tight span of the first triangulation is shown in Figure 5 .

\section{Facets of the SECONDARY POLYTope}

The facets of the secondary polytope correspond to the coarsest regular polyhedral subdivisions $\Pi$. Here coarsest means that $\Pi$ refines no other proper subdivisions. We computed all such subdivisions for the 4-cube. This result gives an irredundant inequality representation of the secondary polytope $\mathcal{N}\left(E_{2222}\right)$ of the 4-cube, analogous to that of the 3-cube in expression (2). 
Since the Newton polytope $\mathcal{N}\left(D_{2222}\right)$ is a summand of the secondary polytope $\mathcal{N}\left(E_{2222}\right)$, all facet normals of $\mathcal{N}\left(D_{2222}\right)$ are also facet normals of $\mathcal{N}\left(E_{2222}\right)$. Thus we already know 268 facets of the secondary polytope from Section 4 . Furthermore the linear equations $A x=(120,60,60,60,60)^{T}$ hold on $\mathcal{N}\left(E_{2222}\right)$.

In order to compute the facets of the secondary polytope of the 4-cube, we first wrote a program to compute the GKZ vector of each regular triangulation of the 4-cube. The information about the triangulations themselves was obtained using TOPCOM. We then used the following method to compute the facets of the secondary polytope, which can be applied to any point configuration with symmetry.

Since $\mathcal{N}\left(E_{2222}\right)$ is an 11-dimensional polytope with 87959448 vertices, computing its facets is currently out of the scope of any general polyhedral software. Thus, to compute the facets, we took advantage of the relationship between the secondary polytope and subdivisions of the 4-cube. Namely, we know that each vertex of $\mathcal{N}\left(E_{2222}\right)$ corresponds to a regular triangulation of the 4-cube, and, by [12, §7.2.C], each edge $(v, w)$ of $\mathcal{N}\left(E_{2222}\right)$ corresponds to the circuit connecting the two triangulations represented by the vertices $v$ and $w$.

Note that, given any triangulation of the 4-cube, there are relatively few circuits which contain a face of the triangulation. As a result, each tangent cone of $\mathcal{N}\left(E_{2222}\right)$ is generated by relatively few vectors. Hence we computed the facets of $\mathcal{N}\left(E_{2222}\right)$ by equivalently computing the facets of its tangent cones. Although there are 87959448 tangent cones, they come in 235227 orbits under the $B_{4}$-action. Thus, we only computed the facets of 235227 tangent cones; all other facets of $\mathcal{N}\left(E_{2222}\right)$ were obtained by applying the $B_{4}$-action.

We found that $\mathcal{N}\left(E_{2222}\right)$ has 80876 facets in 334 orbits. Thus the 4 -cube admits exactly 80876 coarsest regular subdivisions. The distribution of the types of coarsest regular subdivisions according to orbit size is

$$
\begin{array}{cccccccccc}
8 & 12 & 16 & 24 & 32 & 48 & 64 & 96 & 192 & 384 \\
2 & 1 & 4 & 2 & 4 & 14 & 16 & 26 & 132 & 133
\end{array}
$$

Example 19 (The most symmetric coarsest regular subdivisions). The three most symmetric coarsest regular subdivisions come from facets of $\mathcal{N}\left(D_{2222}\right)$. The two coarsest subdivisions with orbit size 8 correspond to Facet 3 and Facet 8 in Sections 4 and 5. Their tight spans are a segment and an octahedron, respectively. The unique subdivision with orbit size 12 corresponds to Facet 2 in Sections 4 and 5 . Its tight span is also a line segment.

The distribution of the orbits according to the number of maximal cells in the subdivision and the dimensions of maximal faces of the tight span are shown in Table 6. A coarsest subdivision of the 4-cube can have up to 13 maximal cells. Here it is no longer the case that the number of vertices and 3 -faces determine the $f$-vector of the tight span. There are 33 types of $f$-vectors.

Example 20 (The missing split). The 4-cube admits 4 splits, i.e., subdivisions obtained by slicing the 4-cube with a hyperplane, whose tight spans are line segments. Three of them correspond to Facets 1,2 , and 3 of $\mathcal{N}\left(D_{2222}\right)$. The remaining split subdivides the 4-cube into 2 cells with 8 and 14 vertices each, having normalized volumes 4 and 20, respectively. The corresponding facets of $\mathcal{N}\left(E_{2222}\right)$ are given by inequalities of the form $x_{0000}+x_{0001} \geq 5$. 
TABLE 6. Distribution of the 334 types of coarsest regular subdivisions. The rows correspond to the number of maximal cells and the columns correspond to the signatures of the tight span.

\begin{tabular}{|c|c|c|c|c|c|}
\hline & $\{3\}$ & $\{3,2\}$ & $\{2\}$ & $\{1\}$ & total \\
\hline 2 & & & & 4 & 4 \\
3 & & & 5 & & 5 \\
4 & 3 & & 7 & & 10 \\
5 & 4 & 3 & 17 & & 24 \\
6 & 5 & 14 & 12 & & 31 \\
7 & 13 & 21 & 39 & & 73 \\
8 & 7 & 31 & 51 & & 89 \\
9 & 7 & 26 & 24 & & 57 \\
10 & 2 & 14 & 21 & & 37 \\
11 & 1 & 1 & & & 2 \\
12 & 1 & & & & 1 \\
13 & 1 & & & & 1 \\
\hline total & 44 & 176 & 110 & 4 & 334 \\
\hline
\end{tabular}

Example 21 (The largest tight span). There is a unique coarsest regular subdivision with 13 maximal cells. Its tight span is a 3-dimensional polytope with $f$-vector $(13,24,13,1)$, as shown in Figure 6 The corresponding facets are given by inequalities like $x_{0000}+x_{1111}-x_{0011} \leq 44$.

Example 22 (The largest tight span with non-pure dimension). Almost half of the coarsest regular subdivisions have a pure dimensional tight span. The largest non-pure dimensional tight span has $f$-vector $(11,20,11,1)$. It contains one 3 dimensional face and two maximal 2-faces. A figure is depicted in Figure 6. The corresponding facets are given by inequalities like

$$
x_{0000}+x_{1010}+x_{1111}-x_{1000}-x_{1001}-x_{1011} \leq 47 .
$$

All the 334 facet inequalities and all the data we have discussed in this paper are available on our website bio.math.berkeley.edu/4cube/.

$(4,8) \quad(20,14)$
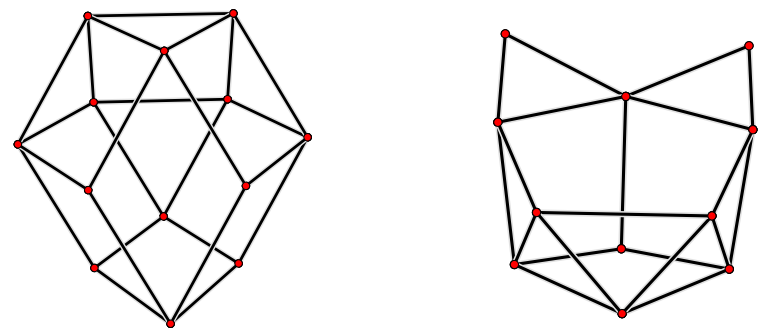

Figure 6. The tight spans for Examples 20, 21, and 22 


\section{ACKNOWLEDGEMENTS}

This work began during Debbie Yuster's fall 2005 visit to the UC Berkeley Mathematics Department. Peter Huggins was supported by an ARCS Foundation Fellowship. Bernd Sturmfels was partially supported by the National Science Foundation (DMS-0456960), and Josephine Yu was supported by an NSF Graduate Research

Fellowship. We also thank Francisco Santos for helpful discussions and comments.

\section{REFERENCES}

[1] E. Allman and J. Rhodes: Phylogenetic invariants for stationary base composition, Journal Symbolic Computation 41 (2006) 138-150. MR2197150 (2006j:92041)

[2] F. Block and J. Yu: Tropical convexity via cellular resolutions, Journal of Algebraic Combinatorics 24 (2006) 103-114. MR2245783 (2007f:52041)

[3] R. W. Cottle: Minimal triangulation of the 4-cube, Discrete Math. 40 (1982) 25-29. MR676709 (84d:05065a)

[4] A. Conca, S. Hosten and R. Thomas: Nice initial complexes of some classical ideals, Algebraic and geometric combinatorics, Contemp. Math., 423, Amer. Math. Soc., Providence, RI, 2006, pp. 11-42. MR2298753

[5] D. Cox, J. Little and D. O'Shea: Using Algebraic Geometry, Graduate Texts in Mathematics 185, second edition, Springer Verlag, New York, 2005. MR2122859 (2005i:13037)

[6] J. De Loera: Nonregular triangulations of products of simplices, Discrete and Computational Geometry 15 (1996) 253-264. MR1380393 (97i:52017)

[7] V. de Silva and L.-H. Lim: Tensor rank and the ill-posedness of the best low-rank approximation problem, SIAM Journal on Matrix Analysis and Applications, to appear.

[8] A. Dickenstein, E.M. Feichtner and B. Sturmfels: Tropical discriminants, Journal of the American Mathematical Society, 20 (2007) 1111-1133.

[9] M. Develin and B. Sturmfels: Tropical convexity, Documenta Math. 9 (2004) 1-27. MR2054977 (2005i:52010)

[10] J. De Loera, J. Rambau and F. Santos: Triangulations: Applications, Structures and Algorithms, Algorithms and Computation in Mathematics, Springer Verlag, Heidelberg, to appear.

[11] E. Gawrilow and M. Joswig: Polymake: a framework for analyzing convex polytopes, in Polytopes - Combinatorics and Computation, eds. G. Kalai and G.M. Ziegler, Birkhäuser, 2000, pp. 43-74. MR1785292 (2001f:52033)

[12] I.M. Gel'fand, M.M. Kapranov, A.V. Zelevinsky: Discriminants, Resultants, and Multidimensional Determinants; Birkhäuser, Boston, MA, 1994. MR1264417 (95e:14045)

[13] H. Hirai: A geometric study of the split decomposition, Discrete and Computational Geometry 36 (2006) 331-361. MR 2252108 (2007f:52025)

[14] P. Lévay: On the geometry of a class of $N$-qubit entanglement monotones, Journal of Physics A 38 (2005) 9075-9085. MR2185885 (2006m:81070)

[15] J.-G. Luque and J.-Y. Thibon: The polynomial invariants of four qubits, Phys. Rev. A 67, 042303 (2003). MR2039690 (2004k:81098)

[16] J. Pfeifle and J. Rambau: Computing triangulations using oriented matroids, in Algebra, Geometry and Software Systems (M. Joswig and N. Takayama, eds.) Springer Verlag, 2003, pp. 49-76. MR2011753 (2004i:68233)

[17] J. Rambau: TOPCOM: triangulations of point configurations and oriented matroids. Mathematical Software (Beijing, 2002), World Sci. Publishing, River Edge, NJ, 2002, 330-340. MR.1932619

[18] F. Santos: The Cayley trick and triangulations of products of simplices, In Integer Points in Polyhedra - Geometry, Number Theory, Algebra, Optimization, (eds. A. Barvinok, M. Beck, C. Haase, B. Reznick, and V. Welker), Contemporary Mathematics 374, Amer. Math. Soc., Providence, 2005. MR2134757 (2005j:00018)

[19] B. Sturmfels and S. Sullivant: Combinatorial secant varieties, Quarterly Journal of Pure and Applied Mathematics, 2 (2006) 285-309, (Special issue: In Honor of Robert MacPherson). MR2252121

[20] B. Sturmfels and J. Yu: Classification of six-point metrics, Electronic Journal of Combinatorics 11 (2004/05) R44. MR2097310 (2005m:51016) 
[21] J.G. Sumner and P.D. Jarvis: Using the tangle: a consistent construction of phylogenetic distance matrices for quartets, Math. Biosci. 204 (2006) 49-67. MR2269665 (2007g:92057)

[22] J.M.F. ten Berge: Kruskal's polynomial for $2 \times 2 \times 2$-arrays and a generalization to $2 \times n \times n$ arrays, Psychometrika 58 (1991) 631-636.

Department of Mathematics, University of California, Berkeley, California 94720

E-mail address: phuggins@math.berkeley.edu

Department of Mathematics, University of California, Berkeley, California 94720

E-mail address: bernd@math.berkeley.edu

Department of Mathematics, University of California, Berkeley, California 94720

Current address: Department of Mathematics, Massachusetts Institute of Technology, Cambridge, Massachusetts 02139

E-mail address: jyu@math.mit.edu

Department of Mathematics, Columbia University, New York, New York 10027

Current address: DIMACS Center, Rutgers University, Piscataway, New Jersey 08854

E-mail address: yuster@math.rutgers.edu 